\title{
Investigation of the thermal and optical performance of a Spatial Light Modulator with high average power picosecond laser exposure for Materials Processing Applications
}

DOI:

10.1088/1361-6463/aaa948

\section{Document Version}

Accepted author manuscript

Link to publication record in Manchester Research Explorer

\section{Citation for published version (APA):}

Zhu, G., Whitehead, D., Perrie, W., Allegre, O., Volle, V., Li, Q., Tang, Y., Dawson, K., Jin, Y., Edwardson, S., Li, L., \& Dearden, G. (2018). Investigation of the thermal and optical performance of a Spatial Light Modulator with high average power picosecond laser exposure for Materials Processing Applications. Journal of Physics D: Applied Physics. https://doi.org/10.1088/1361-6463/aaa948

\section{Published in:}

Journal of Physics D: Applied Physics

\section{Citing this paper}

Please note that where the full-text provided on Manchester Research Explorer is the Author Accepted Manuscript or Proof version this may differ from the final Published version. If citing, it is advised that you check and use the publisher's definitive version.

\section{General rights}

Copyright and moral rights for the publications made accessible in the Research Explorer are retained by the authors and/or other copyright owners and it is a condition of accessing publications that users recognise and abide by the legal requirements associated with these rights.

\section{Takedown policy}

If you believe that this document breaches copyright please refer to the University of Manchester's Takedown Procedures [http://man.ac.uk/04Y6Bo] or contact uml.scholarlycommunications@manchester.ac.uk providing relevant details, so we can investigate your claim.

\section{OPEN ACCESS}


ACCEPTED MANUSCRIPT

\section{Investigation of the thermal and optical performance of a Spatial Light Modulator with high average power picosecond laser exposure for Materials Processing Applications}

To cite this article before publication: Guangyu Zhu et al 2018 J. Phys. D: Appl. Phys. in press https://doi.org/10.1088/1361-6463/aaa948

\section{Manuscript version: Accepted Manuscript}

Accepted Manuscript is "the version of the article accepted for publication including all changes made as a result of the peer review process, and which may also include the addition to the article by IOP Publishing of a header, an article ID, a cover sheet and/or an 'Accepted

Manuscript' watermark, but excluding any other editing, typesetting or other changes made by IOP Publishing and/or its licensors"

This Accepted Manuscript is (C) 2018 IOP Publishing Ltd.

During the embargo period (the 12 month period from the publication of the Version of Record of this article), the Accepted Manuscript is fully protected by copyright and cannot be reused or reposted elsewhere.

As the Version of Record of this article is going to be / has been published on a subscription basis, this Accepted Manuscript is available for reuse under a CC BY-NC-ND 3.0 licence after the 12 month embargo period.

After the embargo period, everyone is permitted to use copy and redistribute this article for non-commercial purposes only, provided that they adhere to all the terms of the licence https://creativecommons.org/licences/by-nc-nd/3.0

Although reasonable endeavours have been taken to obtain all necessary permissions from third parties to include their copyrighted content within this article, their full citation and copyright line may not be present in this Accepted Manuscript version. Before using any content from this article, please refer to the Version of Record on IOPscience once published for full citation and copyright details, as permissions will likely be required. All third party content is fully copyright protected, unless specifically stated otherwise in the figure caption in the Version of Record.

View the article online for updates and enhancements. 


\title{
Investigation of the thermal and optical performance of a Spatial Light Modulator with high average power picosecond laser exposure for Materials Processing Applications
}

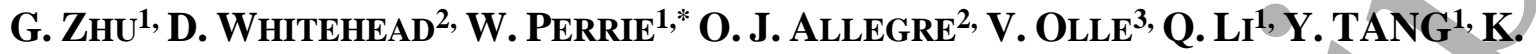 \\ DAWSON $^{4}$, Y. JIN ${ }^{1,}$ S. P. EDWARDSON ${ }^{1}$, L. LI $^{2}$ AND G. DEARDEN ${ }^{1}$ \\ ${ }^{1}$ Laser Group, School of Engineering, Brownlow Street, University of Liverpool, L69 3GQ, UK \\ ${ }^{2}$ Laser Processing Research Centre, School of Mechanical, Aerospace and Civil Engineering, The University of Manchester, Manchester \\ M13 9PL, UK \\ ${ }^{3}$ Hamamatsu Photonics UK Ltd., 2 Howard Court, Welwyn Garden City, Hertfordshire AL7 1BW, UKK \\ ${ }^{4}$ Nanoinvestigation Centre at Liverpool, University of Liverpool, Liverpool, L69 3GL, UK
}

*wpfemto1@liverpool.ac.uk

\begin{abstract}
Spatial light modulators (SLM's) addressed with Computer Generated Holograms (CGH's) can create structured light fields on demand when an incident laser beam is diffracted by a phase CGH. The power handling limitations of these devices based on a liquid crystal layer has always been of some concern. With careful engineering of chip thermal management, we report the detailed optical phase and temperature response of a liquid cooled SLM exposed to picosecond laser powers up to $\langle\mathrm{P}\rangle=220 \mathrm{~W}$ at $1064 \mathrm{~nm}$. This information is critical for determining device performance at high laser powers. SLM chip temperature rose linearly with incident laser exposure, increasing by only $5^{\circ} \mathrm{C}$ at $\langle\mathrm{P}\rangle=220 \mathrm{~W}$ incident power, measured with a thermal imaging camera. Thermal response time with continuous exposure was 1-2 seconds. The optical phase response with incident power approaches $2 \pi$ radians with average power up to $\langle\mathrm{P}\rangle=130 \mathrm{~W}$, hence the operational limit, while above this power, liquid crystal thickness variations limit phase response to just over $\pi$ radians. Modelling of the thermal and phase response with exposure is also presented, supporting experimental observations well. These remarkable performance characteristics show that liquid crystal based SLM technology is highly robust when efficiently cooled. High speed, multi-beam plasmonic surface micro-structuring at a rate $R$ $=8 \mathrm{~cm}^{2} \mathrm{~s}^{-1}$ is achieved on polished metal surfaces at $\langle\mathrm{P}\rangle=25 \mathrm{~W}$ exposure while diffractive, multi-beam surface ablation with average power $\langle\mathrm{P}\rangle=100 \mathrm{~W}$ on stainless steel is demonstrated with ablation rate of $\sim 4 \mathrm{~mm}^{3} \mathrm{~min}^{-1}$. However, above $130 \mathrm{~W}$, first order diffraction efficiency drops significantly in accord with the observed operational limit. Continuous exposure for a period of 45 minutes at a laser power of $\langle\mathrm{P}\rangle=160 \mathrm{~W}$ did not result in any detectable drop in diffraction efficiency, confirmed afterwards by the efficient parallel beam processing at $\langle\mathrm{P}\rangle=100 \mathrm{~W}$. Hence, no permanent changes in SLM phase response characteristics have been detected. This research work will help to accelerate the use of liquid crystal Spatial light modulators for both scientific and ultra high throughput laser-materials micro-structuring applications.
\end{abstract}

\section{Introduction}

The construction of the first $50 \times 50$ pixel Spatial Light Modulator (SLM) based on an active silicon backplane combined with a nematic liquid crystal as the light modulator was described in 1989 by McKnight [1], while development of a 256 x256 array followed within 5 years [2] and commercial development of liquid crystal SLMs has been rapid ever since by companies such as Holoeye (Germany) Boulder Systems (USA) and Hamamatsu (Japan). SLM's have been used for a wide range of both scientific and industrial applications such as wavefront correction in astronomy [3], the creation of spiral laser beams carrying Orbital Angular Momentum (OAM) [4], clarifying the relationship of OAM to Spin Angular Momentum (SAM) [5], static and dynamic parallel beam processing with ultrafast lasers [6-9] and dynamic polarisation control for surface micro-patterning [10]. Also, a competing technology, termed a Grating Light Valve (GLV) is a diffractive Micro-Opto ElectroMechanical System (MOEMS) spatial light modulator, capable of high bandwidth $(\mathrm{kHz})$ modulation of light with applications in high-resolution displays and computer-to-plate printing [10]. The average laser power handling capability of these devices is impressive, around 60W. While in general, these devices can modulate only intensity, recent research using a MOEMS device combined with polarising crystals demonstrated high bandwidth vector field modulation at $5 \mathrm{kHz}[11]$. 
Fixed Diffractive Optic Elements (DOEs) based on precision etched surface profiles on fused silica are very robust, able to handle 10's of Watts but designed and fabricated for one particular function only, whether beam shaping or generating multi beams [12]. SLM's on the other hand, are dynamic diffractive optics with wide flexibility for structuring laser intensity and polarization when applied with appropriate Computer Generated Holograms (CGH's), calculated from inverse Fourier Transforms [13].

Power handling, (both average and peak power) with a liquid crystal based SLM has induced some anxiety with regard to likely damage levels and thus experiments carried out at high power levels have been limited. The first demonstration in this direction was by Beck et al [14] who cooled a commercial, Holoeye SLM, (model LCR-2500) rated for average power $\langle\mathrm{P}\rangle \sim 3 \mathrm{~W}$ maximum power, increasing this well beyond the commercial limit to $\langle\mathrm{P}\rangle=14.8 \mathrm{~W}$ with $532 \mathrm{~nm}$, nanosecond laser pulses. This was achieved by mounting the chip to a large, optically flat copper cooling block. The surface profile of the chip was measured in a stepping Michelson interferometer with the SLM placed in one arm and a flat mirror in the reference arm with the resulting interference pattern observed on a screen by a CCD camera. By converting the phase map (fringes) to height differences, the surface profile of the SLM display before and after mounting onto the flat copper heat sink was determined experimentally. These measurements demonstrated that mounting the CMOS chip altered the surface flatness significantly and by tensioning mounting screws to the copper plate, central flatness was reduced to $\mathrm{R}_{\mathrm{a}} \leq 0.20 \mu \mathrm{m}$ rms at $532 \mathrm{~nm}$. Processing with $532 \mathrm{~nm}$ radiation was then demonstrated with a $4 \mathrm{f}$ system and f-theta focusing lens to a fixed workpiece, imaging a series of complex kinoforms (calculated by IFT's) on metal coated glass (100ms exposure) and shorter, $3.2 \mathrm{~ms}$ exposures on polyimide coated metal with $2 \mathrm{D}$ patterns well defined.

More recently, a pulsed fibre laser $(1065 \mathrm{~nm} / 20 \mathrm{kHz} / 1 \mathrm{~mJ})$ with $\langle\mathrm{P}\rangle=50 \mathrm{~W}$ was integrated with a cooled SLM (Hamamatsu X-11840-03) and galvo scanner for high speed multi- beam surface patterning [15]. Phase CGHs calculated from IFTs defocused the zero order well away from the 10 first order spots and clear, uniform spot letter patterns were ablated on silicon at a rate of $6.10^{5}$ spots per second by the SLM and fast galvo scanner. The same group extended their experiments to cutting of thin, $0.5 \mathrm{~mm}$ thick steel sheets with a CW fibre laser $(1070 \mathrm{~nm})$ operating with $\langle\mathrm{P}\rangle=190 \mathrm{~W}$ continuous exposure [16]. The cutting quality (with reduced burr) was slightly improved with the addition of the low intensity first order spots around the reflected high intensity zero order beam. As the fibre laser beam was randomly polarised, the diffraction efficiency was poor, reaching only 10\%, estimated from the measured intensity profiles.

Very recently, Klerks and Eifel (Pulsar Photonics GmbH) demonstrated laser processing with up to $\langle\mathrm{P}\rangle=60 \mathrm{~W}(404 \mathrm{kHz} / 6 \mathrm{ps})$ of laser radiation at $515 \mathrm{~nm}$ on a cooled SLM in a fully integrated flexible beam shaping system with active cooling of the SLM chip [17]. Phase response measurements were inferred by exposing the SLM (Hamamatsu X13139-04, 1280x1024 pixels) simultaneously to the high average power ps heating laser and an expanded laser pointer, which were overlapped on the SLM. The HP laser was reflected to a beam dump while the laser pointer was directed via a $4 \mathrm{f}$ optical system to the input of a scanning galvo with f-theta lens and imaged to a camera below. Using an integrated temperature chip, active feedback kept the liquid crystal at $14{ }^{\circ} \mathrm{C}$ while laser power was altered over the range $\langle\mathrm{P}\rangle=0-60 \mathrm{~W}$. After each $1 \mathrm{~W}$ power step the grey level required for a phase change of $\pi$ was determined, the so called control parameter. This was measured by applying binary gratings to 180 by 180 pixel regions and altering phase to maximise the \pm 1 order intensities relative to the zero order intensity observed on the camera system below the f-theta lens. When $\mathrm{I}_{ \pm 1} / \mathrm{I}_{0}$ was maximised, the grey level $(\mathrm{GL})$ for a $\pi$ phase change $(\mathrm{GL}=100)$ on a particular region was determined and found to vary little over the full $60 \mathrm{~W}$ exposure range. Robust parallel beam surface micromachining was then demonstrated on stainless steel with powers from $\langle\mathrm{P}\rangle=15 \mathrm{~W}$ to $60 \mathrm{~W}$. At $\langle\mathrm{P}\rangle=60 \mathrm{~W}$ exposure, the company logo (created using a complex kinoform) was also micro-machined cleanly but there was also evidence of detrimental effects due to lower intensity ghost beams on the surface.

Our experience over a decade with the X-10468 (Hamamatsu) series of reflective phase only SLMs showed that peak intensities $>10 \mathrm{GW} / \mathrm{cm}^{2}$ and average power $\langle\mathrm{P}\rangle \sim 12 \mathrm{~W}$ without cooling led to no detectable deterioration after years of continuous operation. These are impressive characteristics. However, the idea of pushing these devices to much higher exposures certainly induced anxiety, based on the likely cost of chip replacement in the event of thermal damage. The perceived limitations on robustness of the liquid crystal layer, particularly with average power exposure has, until now, severely limited industrial uptake of this technology, particularly for laser-materials processing.

In this paper, we first investigated the thermal response of cooled liquid crystal on Silicon (LCOS) SLMs with picosecond laser exposure up to $\langle\mathrm{P}\rangle=220 \mathrm{~W}$. Then, the complete phase response was measured

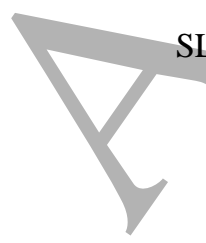


with incident power to ascertain device performance at high powers. Finally, with this knowledge, diffractive multi-beam materials processing was demonstrated with up to $\langle\mathrm{P}\rangle=250 \mathrm{~W}$ laser power.

\section{Method and Experimental details}

Two separate experimental set-ups were created for measuring chip temperature and phase response respectively with laser exposure. For chip temperature measurements, a simple set-up, shown in the schematic of Figure 1(a) was used. The output beam from an Edgewave, Innoslab laser, (10ps, 1064nm, 2MHz) Model PX400-3-GH, located above an optical table was directed downwards by a $90^{\circ}$ turning periscope which brought the beam to required level for the SLM, a Hamamatsu X13139-03, with 1280 x 1024pixels, $12.5 \mu \mathrm{m}$ pixel size and $16 \mathrm{x}$ $12 \mathrm{~mm}$ dimension. The beam was then expanded with a telescope ( $\mathrm{x} 4, \mathrm{f}_{1}=-50 \mathrm{~mm}, \mathrm{f}_{2}=200 \mathrm{~mm}$, AR coated) before reflecting from the SLM at low angle of incidence AOI $<10^{\circ}$ and directed to an air cooled power meter (Gentec UP55G-500F-H12) rated for 500W. A calibrated far infrared thermal camera (model FLIR SC660, sensitive from $8 \mu \mathrm{m}-15 \mu \mathrm{m}$ ) was positioned around $0.5 \mathrm{~m}$ from the SLM for capturing thermal images. It was possible also to take real time videos with this camera.

The SLM was mounted on an engineered copper block, thermally connected to the rear of the silicon chip with copper tubes on the side for liquid cooling of the unit. The block has internally structured cooling channels and the circulating liquid was pumped by a Koolance (model EX2-755) liquid to air cooling unit rated for $500 \mathrm{~W}$. Laser repetition rates of $10 \mathrm{kHz}-2 \mathrm{MHz}$ were employed by pulse selection from the oscillator. Figure 1 (b) shows a photo of the mounted SLM with cooling connectors.

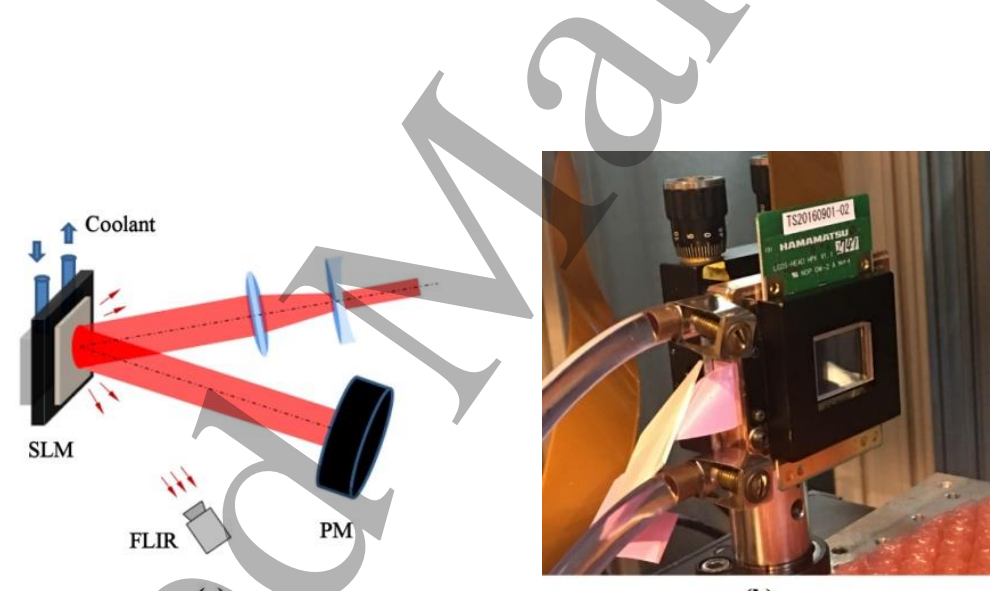

(b)

Figure 1. (a) Schematic of experimental set-up for high power thermal tests of cooled SLM. The raw laser beam was expanded $x 4$, and reflected at low angle from the SLM to the power meter. A thermal camera (FLIR SC660) was positioned to detect heat radiated from the SLM chip and enclosure, (b) photo of cooled SLM (Hamamatsu X13139-03) on mirror mount with liquid cooling connections to the Copper heat sink.

The phase response of the cooled SLM was investigated with the experimental set-up shown in Figure 2. The laser output was expanded $\mathrm{x} 3$ and linear polarisation rotated to $\theta=45^{\circ}$ on the SLM with a half wave plate (Altechna 2-CPW-TZ0-L2-1064). The SLM director was horizontal. A series of CGH's with grey level GL = 0255(8bit) were applied, so that the SLM now behaved as a variable waveplate (introducing a phase delay $\phi$ between the vertical and horizontal electric field components) hence reflected elliptical polarisations. By placing a quarter waveplate (Altechna 2-CPW-TF0-L4-1064) with fast axis at $\theta=45^{\circ}$, reflected elliptical polarisation was re-converted to linear polarisation whose rotation direction $\theta$ depended linearly on the applied grey level CGH. This linear polarisation was then analysed by a thin film polarizer (TFP, Altechna 2-HC45TPF-10640254). The transmitted $|\mathrm{p}\rangle$ and reflected $|\mathrm{s}\rangle$ components were measured with air cooled power meters, Gentec UP55G-500F-H12 (500W) and Gentec UP55N-300F-H12-DO (300W) respectively. A series of phase response 
curves with increasing exposure were measured. The polarisation optics, which are ion beam coated have ultralow low absorption and ultrahigh damage thresholds, quoted as $\mathrm{F}_{\mathrm{th}}>20 \mathrm{Jcm}^{-2}$ with a single ns pulse [18]

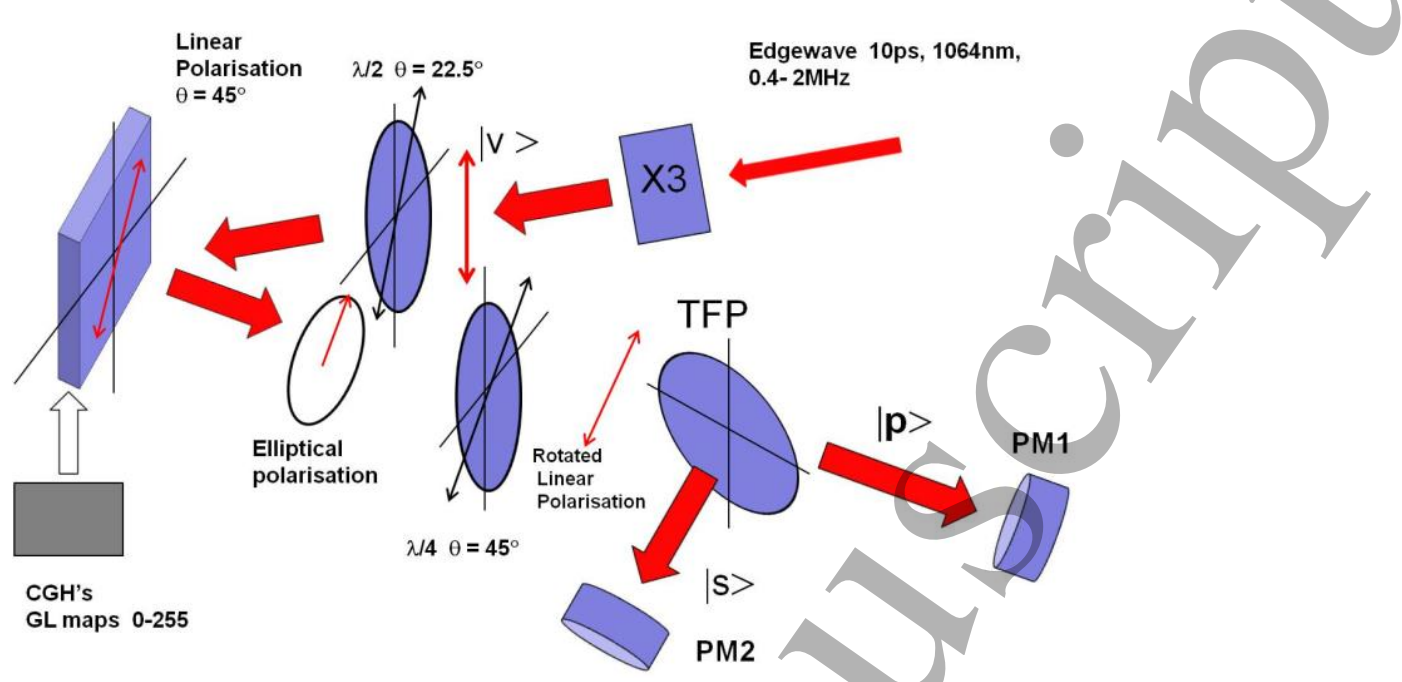

Figure 2. Optical set-up used to determine phase response of cooled SLM using polarisation modulation induced by GL phase maps applied to the SLM. Linear polarisation, incident at $\theta=45^{\circ}$ on the SLM is reflected as elliptical polarisation then converted to linear polarization by the $\lambda / 4$ plate with polarisation direction dependent on the GL phase CGH applied.

Let us assume for the moment that the incident linear polarisation, after reflection from the SLM is right circularly polarised, $|R\rangle=\left[\frac{1}{-i}\right]$ with phase difference $\phi=-\frac{\pi}{2}$ between electric field components. Using the Jones matrix for a $\lambda / 4$ plate tilted at $\theta=45^{\circ}$ the resulting polarisation after traversing the $\lambda / 4$ plate is given by, [19]

$$
|\Psi\rangle=\left[\begin{array}{ll}
1 & \mathrm{i} \\
\mathrm{i} & 1
\end{array}\right]\left[\begin{array}{c}
1 \\
-\mathrm{i}
\end{array}\right]=\left[\begin{array}{l}
2 \\
0
\end{array}\right]=2\left[\begin{array}{l}
1 \\
0
\end{array}\right]=2|\mathrm{x}\rangle
$$

which is linearly polarised in the horizontal direction while the effect on left circularly polarised $|\mathrm{L}\rangle=\left[\frac{1}{\mathrm{i}}\right]_{\text {with }}$ phase difference $\phi=\frac{\pi}{2}$ yields vertically linearly polarised light, $|\mathrm{y}\rangle$. Hence, linear polarisation is rotated though an angle $\Delta \theta=\pi / 2$ while the phase difference between the orthogonal states $|\mathrm{R}\rangle$ and $|\mathrm{L}\rangle, \Delta \phi=\pi$. The rotation angle of linear polarisation after the waveplate is thus given by $\Delta \theta=\Delta \phi / 2$. If the linear polarisation now makes an angle ${ }^{\theta}$ with respect to the transmission axis of the polariser, which is horizontal, the transmitted (reflected) amplitude is given by $E_{0} \cos \theta\left(E_{0} \sin \theta\right)$ while the transmitted (reflected) intensities are given by $I_{0} \cos ^{2} \theta$ $\left(I_{0} \sin ^{2} \theta\right)$ The transmitted (reflected) powers are hence given by $P_{0} \cos ^{2}(\phi / 2),\left(P_{0} \sin ^{2}(\phi / 2)\right)$ where $P_{0}$ is the incident power and $\phi$ is the phase applied to the SLM. This function has two maxima over $2 \pi$ radian. This simple theoretical analysis assumes that the incident laser is highly linearly polarised ( $>100: 1)$ and that the TFP has a high contrast, which is indeed the case, stated to be $T_{p} / T_{s}>1000: 1$ with wavefront error $<\lambda / 8$ in the visible [16]. The blazing function of the SLM, supplied with Hamamatsu software is linear, so that $\varphi \propto$ GL . 


\subsection{Experimental Results}

\subsection{Thermal tests}

Two X-13931-03 Hamamatsu liquid cooled devices were tested. For accurate temperature measurements, the emissivity on the FLIR camera software was set to $\varepsilon=0.73$, the value relevant for Silicon (back plane) near room temperature [20]. When setting $\varepsilon$ instead to 0.93 , (fused silica window), this had only a minor effect on measured absolute temperatures. Figure 3 shows the temperature response of device- 01 with up to $\langle\mathrm{P}\rangle=140 \mathrm{~W}$ exposure, showing that this is highly linear with a maximum temperature rise $\Delta T=5.5^{\circ} \mathrm{C}$ and hence a thermal response coefficient $\mathrm{m}_{01}=0.041^{\circ} \mathrm{C} / \mathrm{W}$, an impressive result. Laboratory ambient temperature was cool early in the morning.

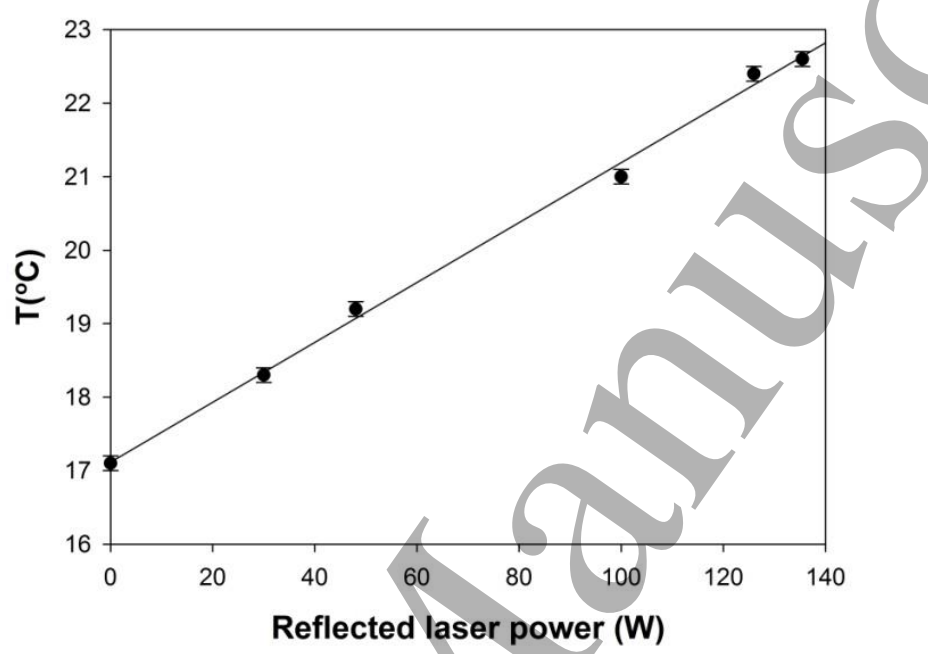

Figure 3. Measured temperature rise with reflected laser power, device- 01 . The thermal response coefficient is measured to be $0.041^{\circ} \mathrm{C} / \mathrm{W}$. Laser repetition rate $\mathrm{f}=404 \mathrm{kHz}$. Errors represent $1 \sigma$ and fit is least squares with high confidence, $\mathrm{R}=0.99$.

Chip temperature response of the liquid crystal device-02 with reflected laser power in the range $\langle\mathrm{P}\rangle=50$ $215 \mathrm{~W}$ is shown in Figure 4. Again, temperature rises linearly with reflected power over this range, however, the temperature difference with $\langle\mathrm{P}\rangle=215 \mathrm{~W}$ (reflected) was only $\Delta T=5.0^{\circ} \mathrm{C}$ while ambient chip temperature was $\mathrm{T}_{0}=21.8^{\circ}$. The response of this cooled SLM is more impressive, yielding a gradient $\mathrm{m}_{01}=0.026^{\circ} \mathrm{C} / \mathrm{W}$ with a cooling efficiency higher by a factor of $\sim 1.6$ over device -01 . As device reflectivity $\mathrm{R}=97 \%$, incident laser power was therefore $\langle\mathrm{P}\rangle=220 \mathrm{~W}$. While these devices are apparently identical models, there is clearly a difference in the thermal cooling efficiency, the source of which is not yet understood. 

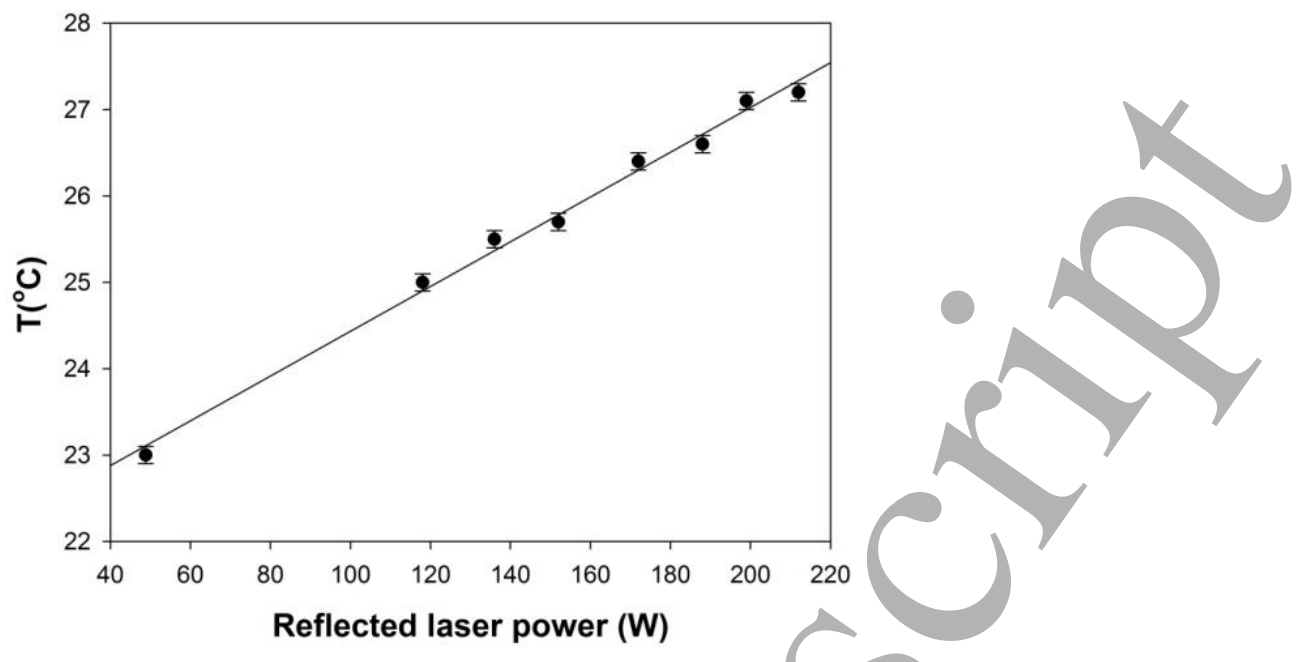

Figure.4 Measured temperature rise with reflected laser power, device-02. Laser repetition $\mathrm{f}=1 \mathrm{MHz}$. Temperature response is excellent with a gradient $\mathrm{m}_{02}=0.026^{\circ} \mathrm{C} / \mathrm{W}$, superior to device -01 . Errors represent $1 \sigma$ and fit is least squares with high confidence, $\mathrm{R}=0.99$

These highly linear characteristics imply that the effective absorption coefficient, $\alpha$ of the chip assembly (window, liquid crystal layer, dielectric reflective layer and silicon backplane) is constant throughout and has only linear temperature dependence in this power range when cooled effectively.

Figure 5 shows typical thermal camera images recorded as laser exposure was increased from $\langle\mathrm{P}\rangle=0$ to $220 \mathrm{~W}$, demonstrating the excellent cooling efficiency of device -02 . Vertically scattered radiation from the incident laser beam warms the surrounding black absorbing SLM enclosure a few degrees, figures 5(b-f). Clear evidence of the intense beam exposure appears in figure $5(\mathrm{e})$ at $\langle\mathrm{P}\rangle=215 \mathrm{~W}$ near the chip centre, a few degrees higher in temperature than the rest of the chip. The high power laser exposure combined with efficient cooling now leads to a temperature gradient across the chip, figures $5(\mathrm{~d}-\mathrm{f})$.
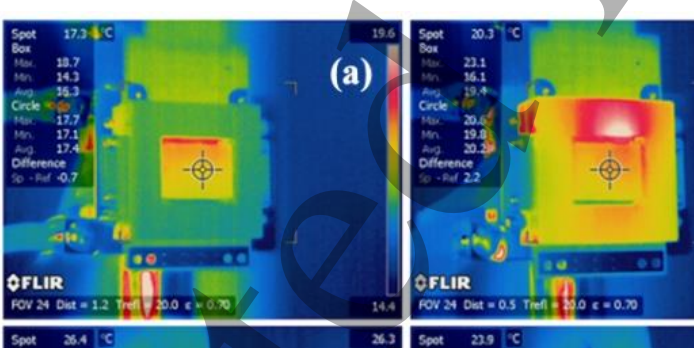

(b)
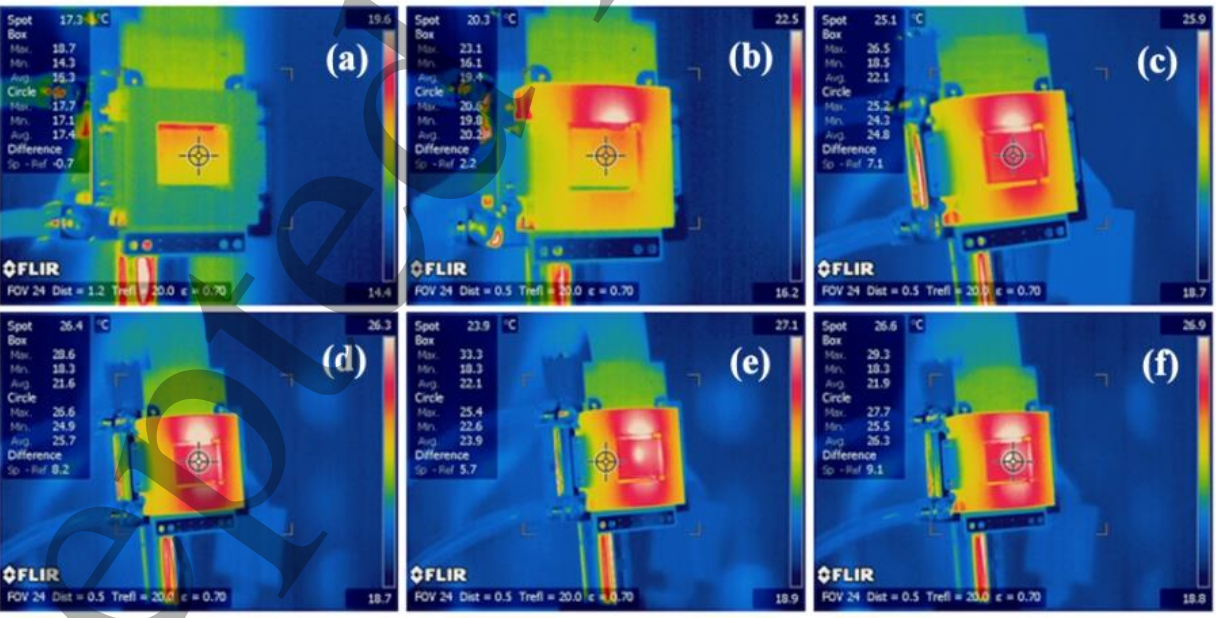

Figure 5. Thermal images of cooled SLM chip with increasing reflected laser exposure (a) $\langle\mathrm{P}\rangle=0$, (b) $\langle\mathrm{P}\rangle=44 \mathrm{~W}$, (c) $\langle\mathrm{P}\rangle=118 \mathrm{~W}$, (d) $\langle\mathrm{P}\rangle=172 \mathrm{~W}(\mathrm{e})\langle\mathrm{P}\rangle=215 \mathrm{~W}$, showing evidence of laser spot near the centre of chip, (f) $\langle\mathrm{P}\rangle=215 \mathrm{~W}$ showing spot temperature $\mathrm{T}=$ $26.6^{\circ} \mathrm{C}$. Note that there was some off axis low intensity scatter from the laser beam reaching the surrounding enclosure (matt black) hence showing a slight temperature rise above and below on this highly absorbing surface. With the scattered radiation removed with an aperture, FLIR images showed a reduced enclosure temperature, generally cooler than the chip temperature. 

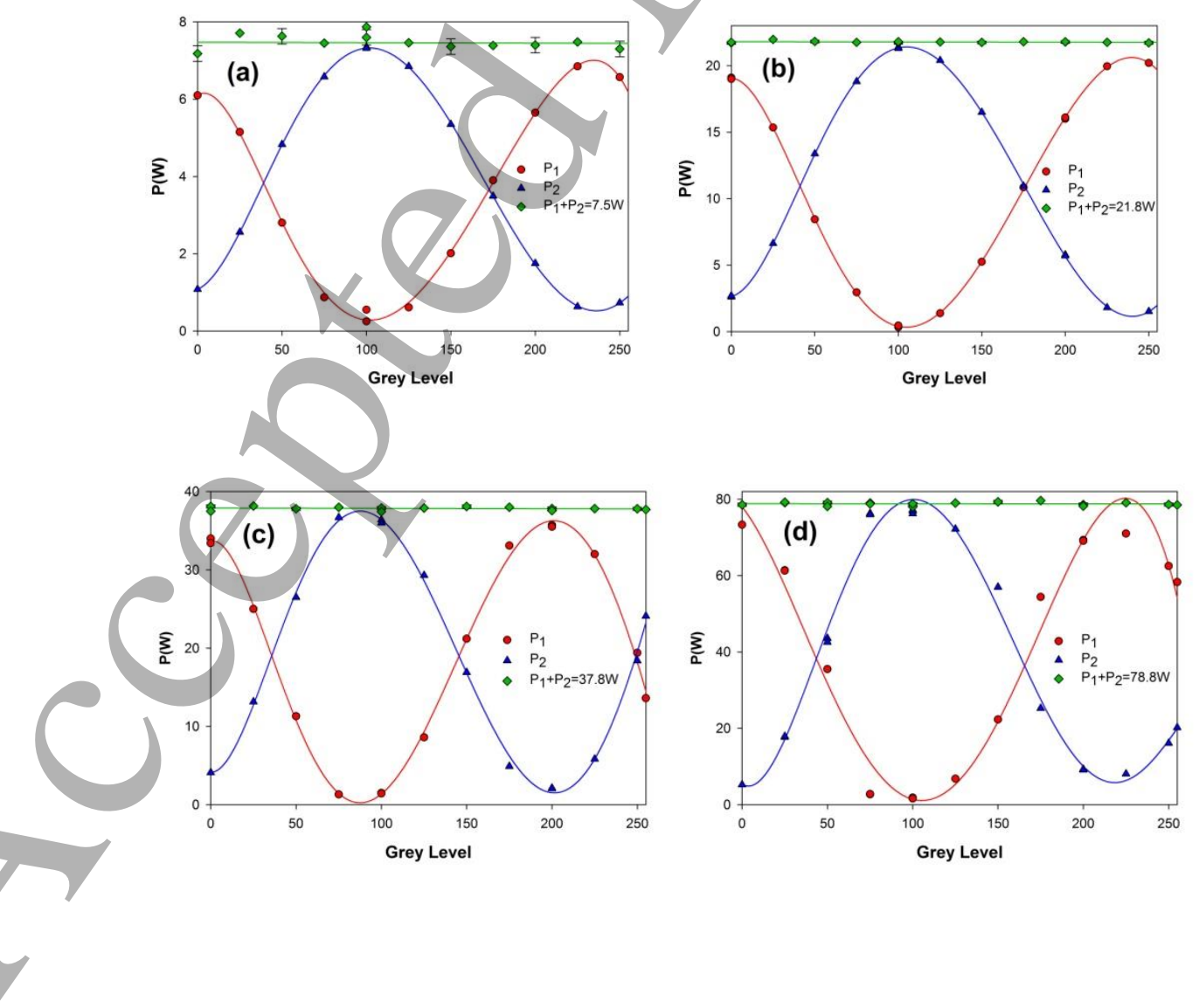

When the laser power at $\langle\mathrm{P}\rangle=220 \mathrm{~W}$ was cut off instantly, the chip temperature cooled back to ambient in approximately 1-2 seconds, supporting the high thermal diffusion rate. With $\mathrm{R}=97 \%$ reflectivity, almost $\langle\mathrm{P}\rangle=$ $7 \mathrm{~W}$ was efficiently removed from the SLM structure. A comparison with our previous work using an uncooled Hamamatsu X10468-04 $(532 \mathrm{~nm})$ at $\langle\mathrm{P}\rangle \sim 8 \mathrm{~W}$ exposure with a Gaussian beam showed a temperature rise $\Delta \mathrm{T}$ $\sim 2^{\circ}$ or a thermal response of $\sim 0.25^{\circ} \mathrm{C} / \mathrm{W}$, thus 10 times poorer than in cooled SLM, device- 02 .

\subsection{Phase measurements}

The phase modulation response of the cooled SLM's, critical for determining SLM performance under intense laser exposure is now presented for the experimental set-up shown above in Figure 2. Measured phase response curves are shown in Figure 6(a-f) for total power $\left\langle\mathrm{P}_{1}+\mathrm{P}_{2}\right\rangle=7.5 \mathrm{~W}, 21.8 \mathrm{~W}, 37.8 \mathrm{~W}, 78.8 \mathrm{~W}, 91.1 \mathrm{~W}$ and 137.5W respectively on device-01. The fits are based on 6 'th order regression polynomials. In figure $6(\mathrm{a})$, at lowest, $7.5 \mathrm{~W}$ exposure, the expected near sinusoidal response with GL is very clear and the sum of powers $\left\langle\mathrm{P}_{1}+\mathrm{P}_{2}\right\rangle$ nearly constant. The error bars show one standard deviation. Where the curves cross, the polarisation incident after reflection from the SLM is circular while at the maxima and minima, linearly polarised with a high degree of polarisation. There is a $\pi$ phase change between the maxima (blue to red), minima, or crossing points. This phase response (apart from a phase shift) follows the predicted $\cos ^{2}(\phi / 2)\left(\sin ^{2}(\phi / 2)\right)$ functions, essentially identical up to $\langle\mathrm{P}\rangle=38.5 \mathrm{~W}$, figure $6(\mathrm{c})$ while at $\langle\mathrm{P}\rangle=79 \mathrm{~W}$ and $\langle\mathrm{P}\rangle=91 \mathrm{~W}$, figure $7(\mathrm{~d})$, figure $7(\mathrm{e})$, there is now a deviation from the ideal phase response near the peaks but phase variation still almost $2 \pi$ at GL $=225$. However, at $\left\langle\mathrm{P}_{1}+\mathrm{P}_{2}\right\rangle=132.5 \mathrm{~W}$ exposure, the response curve has altered significantly, yielding just over a $\pi$ phase change. However, note that at grey level GL $\neq 100$, the reflected polarisation is still highly linear with degree of polarisation $P=\left(I_{\max }-I_{\min }\right) /\left(I_{\max }+I_{\min }\right) \sim 0.93$ and an excellent sign that the liquid crystal layer is not under stress and still functioning. Ofcourse, phase and wavefront errors can be expected on reflected radiation at this laser power. The total exposure time of device -01during these phase tests was approximately 1.5 hours. 

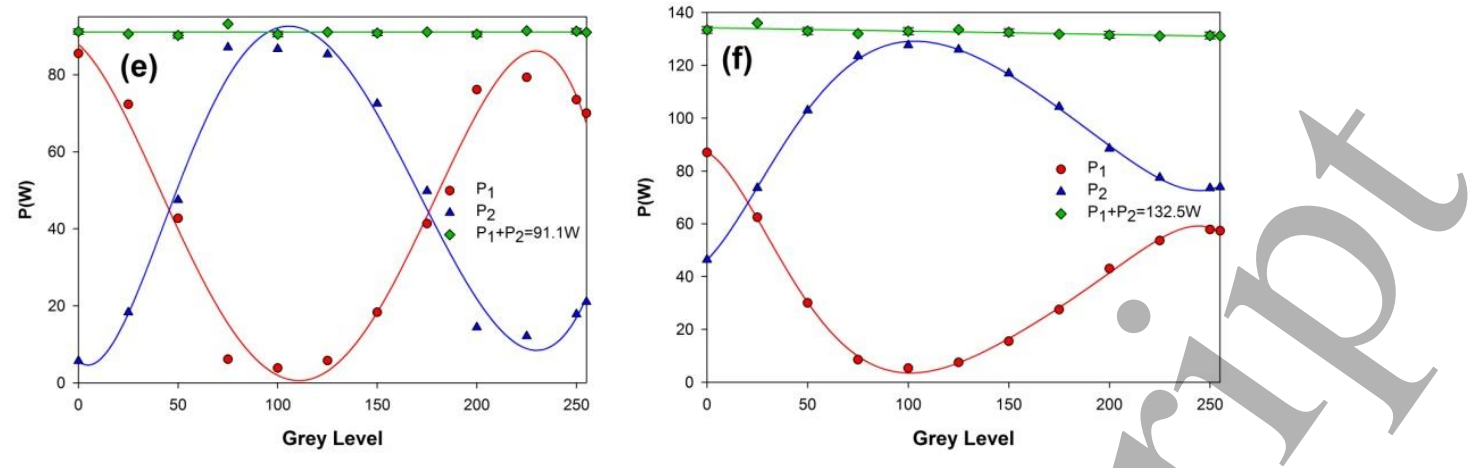

Figure 6. Phase response of cooled SLM -01 (a) $\langle\mathrm{P}\rangle=7.5 \mathrm{~W}$, (b) $\langle\mathrm{P}\rangle=21.8 \mathrm{~W}$, (c) $\langle\mathrm{P}\rangle=37.8 \mathrm{~W}$, (d) $\langle\mathrm{P}\rangle=78.8 \mathrm{~W}$, (e) $\langle\mathrm{P}\rangle=91.1 \mathrm{~W}$ and (f) $\langle\mathrm{P}\rangle=132.5 \mathrm{~W}$. The phase response is very satisfactory up to laser power $\langle\mathrm{P}\rangle=37.8 \mathrm{~W}$ but begins to deviate slightly above $80 \mathrm{~W}$ while a significant deviation occurs at $\langle\mathrm{P}\rangle=132.5 \mathrm{~W}$, reaching just over a $\pi$ phase shift instead of the desired $2 \pi$.

The results of phase response measurements on device -02 are shown in figures $7(\mathrm{a}-\mathrm{e})$ with power levels over the range $\left\langle\mathrm{P}_{1}+\mathrm{P}_{2}\right\rangle=2.6 \mathrm{~W}-196 \mathrm{~W}$. This time, the phase response curves are satisfactory up to $109 \mathrm{~W}$, figure $7(\mathrm{~d})$ achieving a full $2 \pi$ phase change coupled with a high degree of polarisation $P=\left(I_{\max }-I_{\min }\right) /\left(I_{\max }+I_{\min }\right)=0.90$. At $\langle\mathrm{P}\rangle=160 \mathrm{~W}$, figure $7(\mathrm{e})$ and $\langle\mathrm{P}\rangle=196 \mathrm{~W}$, figure $7(\mathrm{f})$, there is reduced phase modulation reaching just over $\pi$ radians while the degree/ of polarisation reduces to $P=$ 0.75 and 0.64 respectively. This drop in observed degree of polarisation is likely due to the temperature gradient across the chip, altering reflected state of polarisation from centre to the beam edge. The changes in response curves infer that wavefront errors will occur during application of phase only CGHs with incident laser powers exceeding $\langle\mathrm{P}\rangle=109 \mathrm{~W}$, altering desired relative intensities in a structured light field. Total exposure time on device- 02 during these measurements was several hours and for 45 minutes at $\langle\mathrm{P}\rangle=$ $160 \mathrm{~W}$ power level. When reducing power levels after exposure at 196W, phase reponses were reproducible.
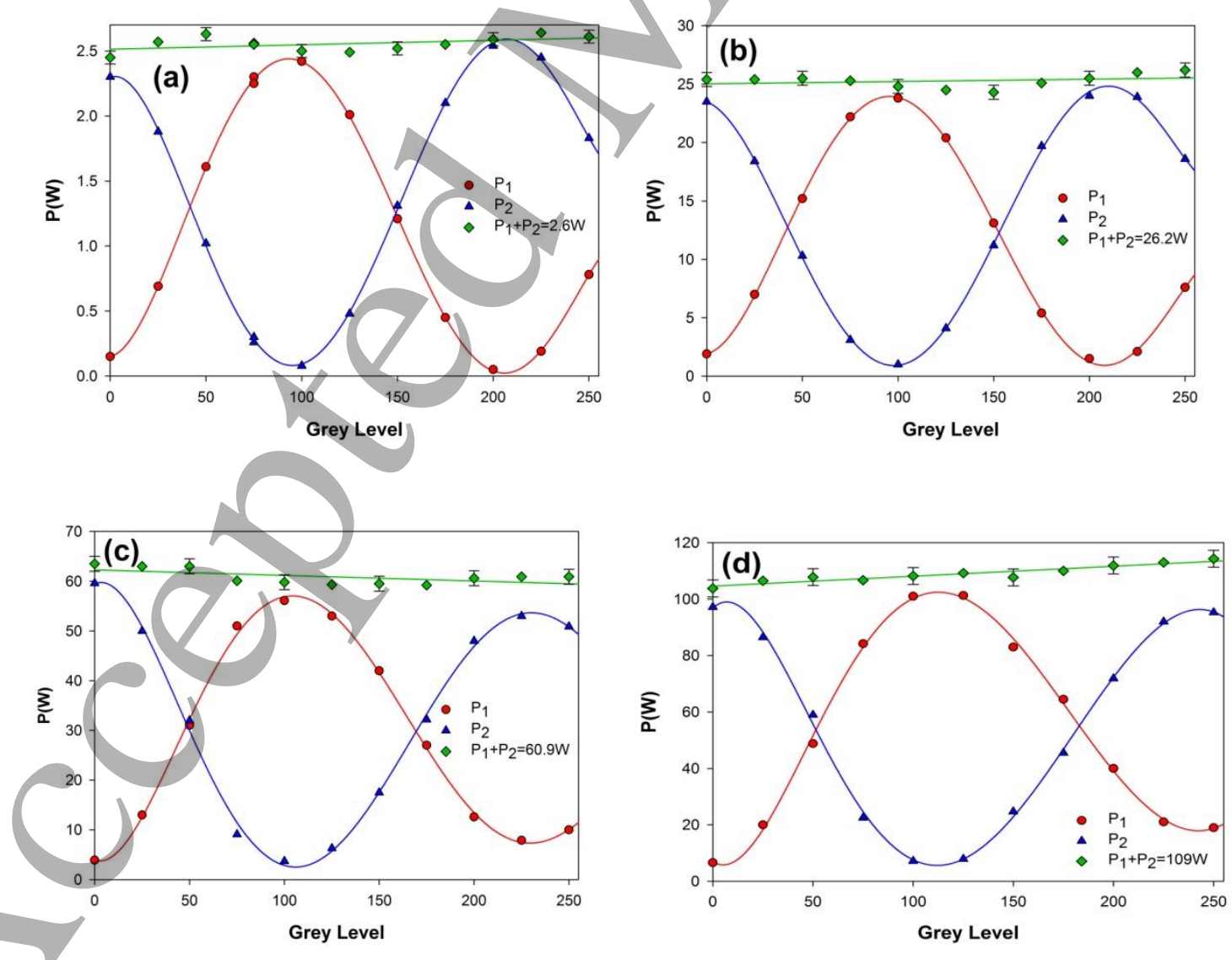


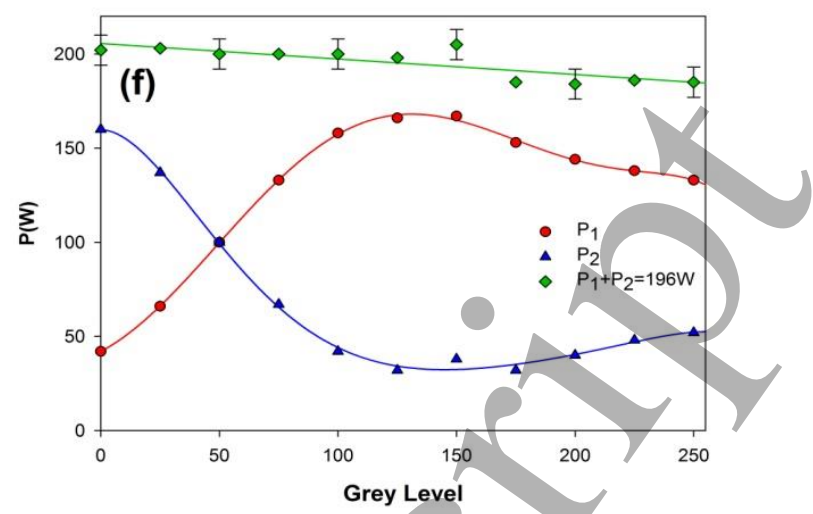

Figure 7. Phase response of cooled SLM -02 (a) $\langle\mathrm{P}\rangle=2.6 \mathrm{~W},(\mathrm{~b}),\langle\mathrm{P}\rangle=26.2 \mathrm{~W}$, (c) $\langle\mathrm{P}\rangle=60.9 \mathrm{~W}$, (d) $\langle\mathrm{P}\rangle=109 \mathrm{~W}$, (e) $\langle\mathrm{P}\rangle=160 \mathrm{~W}$ and $(\mathrm{f})\langle\mathrm{P}\rangle=196 \mathrm{~W}$. The phase response is very satisfactory at laser power up to $\langle\mathrm{P}\rangle=109 \mathrm{~W}$ but deviates significantly at $\langle\mathrm{P}\rangle=$ $160 \mathrm{~W}$ and above, reaching over a $\pi$ phase shift instead of the desired $2 \pi$.

\section{Modelling of observed thermal and phase response}

It is critical to explain the observed temperature and phase response from a theoretical standpoint. However, as exact SLM structure and materials are commercially protected, approximations of these and physical dimensions are made so that modelling may be limited by this lack of complete knowledge.

The silicon chip ( $\sim 1 \mathrm{~mm}$ thick), is connected to a ceramic cooling plate (AlN with similar thermal conductivity of $150 \mathrm{Wm}^{-1} \mathrm{~K}^{-1}$ ) then thermally connected to the water cooled copper heat sink. The liquid crystal (LC) properties, for example, the LC birefringence, its temperature response and nematic-isotropic transition range are unknown, hence approximate values based on the literature of LC data are used to estimate the phase response with temperature.

a) Thermal response

Assume for the moment that absorption in the window (fused silica) and LC layer are neglible. As the dielectric coating reflectivity $\mathrm{R}=97 \%$, then $\langle\mathrm{P}\rangle=7 \mathrm{~W}$ is absorbed in the Silicon for $\langle\mathrm{P}\rangle=220 \mathrm{~W}$ incident average power. We therefore assumed a $7 \mathrm{~W}$ thermal heat source with a Gaussian beam distribution of $10 \mathrm{~mm} 1 / \mathrm{e}^{2}$ diameter. A thermal model using the 3D heat diffusion equation was used in COMSOL Multi-physics software which contains the relevant physical properties such as density, heat capacity and thermal conductivity for each material ( $\mathrm{Si}, \mathrm{AlN}$ and copper). Water cooling of the copper base was also introduced with flow rate $\sim 1 \mathrm{l} / \mathrm{min}$. Appropriate mesh sizes from 1-3mm were used for the numerical calculations.

Theoretical results are shown in figure 8. With no cooling, figure 8(a) shows the calculated temperature rise of the Silicon top surface (red), the Si-ceramic interface (green) and ceramic-Copper interface (blue). The system, as expected, does not come to thermal equilibrium. The surface temperature rises $2^{\circ} \mathrm{C}$ in the first few seconds then increases at a linear rate of $0,024^{\circ} \mathrm{C} / \mathrm{sec}$. Ambient temperature was $293^{\circ} \mathrm{K}$.

The effect of adding water cooling of the bottom copper surface at $20^{\circ} \mathrm{C}$ is shown in figure 8 (b) demonstrating that thermal equilibrium is reached after approximately 20 seconds. The chip temperature rises rapidly in the first second then comes to a stable temperature with a $2^{\circ} \mathrm{C}$ rise. The expected temperatures at the interfaces are also plotted showing that there are temperature gradients throughout, physically realistic. While the absolute temperature rise at the silicon surface is lower than that observed by a factor of 2.5 , nevertheless, this model supports the view that absorption in Silicon is a major source of the observed temperature rise during laser exposure of the SLM. 

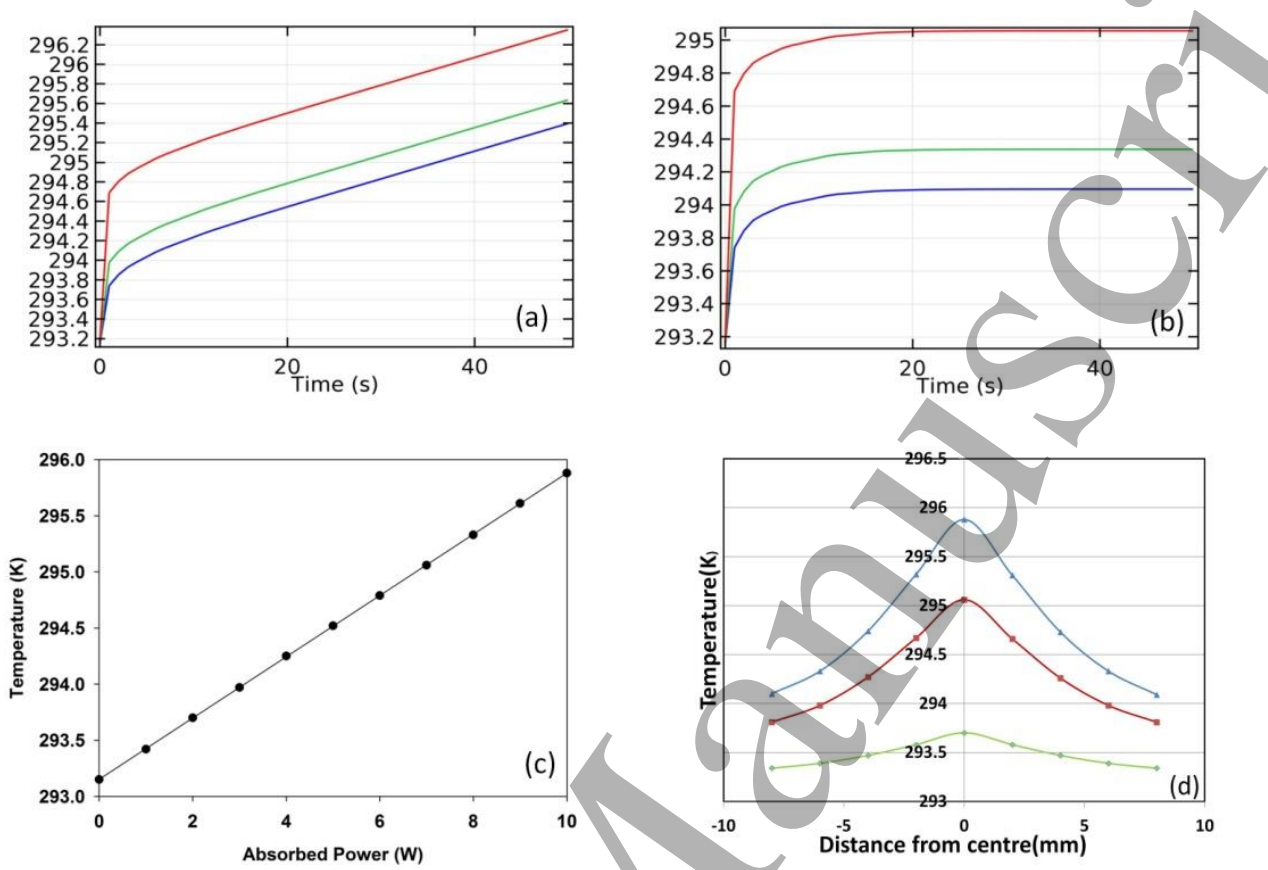

The calculated surface temperature of Silicon with absorbed power $(\langle\mathrm{P}\rangle=0-10 \mathrm{~W})$ is shown in figure $8(\mathrm{c})$, yielding a linear rise with exposure (absorbed power) which is in agreement with experimental observation, (figure 4). The calculated temperature distribution across the chip with exposure is shown in figure 8(d), indicating that a thermal gradient exists across the chip, increasing with exposure in accord with the FLIR camera observations (figure 5).

Figure 8. Expected chip temperature with $\langle\mathrm{P}\rangle=7 \mathrm{~W}$ absorbed $(\langle\mathrm{P}\rangle=220 \mathrm{~W}$ incident), (a) without water cooling of copper heat sink, (b) with water cooling of copper heat sink showing that thermal equilibrium is achieved, (c) chip temperature (centre) with absorbed power, d) Calculated temperature distribution after reaching thermal equilibrium with absorbed powers of $\langle\mathrm{P}\rangle=2 \mathrm{~W}, 7 \mathrm{~W}$ and $10 \mathrm{~W}$ (incident powers of $\langle\mathrm{P}\rangle=63 \mathrm{~W}, 220 \mathrm{~W}$ and $315 \mathrm{~W}$ respectively.)

The window material is likely to be fused silica which has a remarkably low absorption coefficient at $1064 \mathrm{~nm}$, $\alpha_{\mathrm{fs}}^{1064}=1.10^{-5} \mathrm{~cm}^{-1}[21]$ hence we the absorbed power with incident average power $\langle\mathrm{P}\rangle=220 \mathrm{~W}$ is $\langle 1 \mathrm{~mW}$. After a 10 s exposure, the expected temperature rise $\Delta \mathrm{T} \sim 3.6 .10^{-3}{ }^{\circ} \mathrm{C}$, neglible.

The LC, transparent in the visible and NIR, must also have a low absorption coefficient. As such materials often contain organic molecules such as Benzene rings and hydrocarbon end groups, the absorption coefficient of such molecules can be used to estimate a value for the LC. In benzene, $\alpha_{532}=4.10^{-4} \mathrm{~cm}^{-1}$ [22]. At 1064nm, this is likely to be similar. Assuming that $\alpha_{\mathrm{LC}}^{1064} \sim 1.10^{-3} \mathrm{~cm}^{-1}$, a density $\rho \sim 1 \mathrm{gcm}^{-3}$, a specific heat $\mathrm{C} \sim 2.4 \mathrm{Jg}^{-1} \mathrm{~K}^{-1}$, [23] a LC thickness of around $2 \mu \mathrm{m}$, then the absorbed power $\left\langle\mathrm{P}_{\mathrm{ABS}}\right\rangle=\left(1-\mathrm{e}^{-\alpha \mathrm{L}}\right)\left\langle\mathrm{P}_{0}\right\rangle \sim 4.10^{-5} \mathrm{~W}$ and expected temperature rise with a 10 s exposure $\Delta \mathrm{T} \sim 0.4^{\circ} \mathrm{C}$, which is significant. This estimate is sensitive to the actual LC absorption coefficient which may be higher.

(b) Phase Change with exposure.

The LC is a nematic liquid crystal and a thermotropic transition to the isotropic phase will occur at the transition temperature, $\mathrm{T}_{\mathrm{NI}}$ where the LC birefringence collapses. If the LC layer has thickness L and birefringence $\Delta \mathrm{n}$, 
then variations in the optical thickness, $\Delta \mathrm{nL}$ will cause phase changes. After reflection from the dielectric reflecting layer, the phase change is given by $\phi=(4 \pi / \lambda) \Delta \mathrm{nL}$. The total change with temperature is given by,

$$
\frac{\partial \phi}{\partial \mathrm{T}}=(4 \pi / \lambda)\left[\mathrm{L} \frac{\partial(\Delta \mathrm{n})}{\partial \mathrm{T}}+\Delta \mathrm{n} \frac{\partial \mathrm{L}}{\partial \mathrm{T}}\right]
$$

Assuming for the moment that the LC layer thickness is unaffected by temperature, $\mathrm{L}$ is constant so that the second term in equation (1) is zero. The change of phase with temperature is then,

$$
\frac{\partial \phi}{\partial \mathrm{T}}=(4 \pi / \lambda) \frac{\partial(\Delta \mathrm{n})}{\partial \mathrm{T}}
$$

The variation of birefringence with temperature in LC's is well represented by an empirical equation, [24] $\Delta \mathrm{n}=\Delta \mathrm{n}_{0}\left(1-\mathrm{T} / \mathrm{T}_{\mathrm{IN}}\right)^{\beta}$ where $\Delta \mathrm{n}_{0}$ is the birefringence extrapolated to $0^{\circ} \mathrm{K}, \mathrm{T}$ is temperature $\left({ }^{\circ} \mathrm{K}\right), \mathrm{T}_{\mathrm{NI}}$ is the nematic-isotropic phase transition temperature $\left({ }^{\circ} \mathrm{K}\right)$ and the index $\beta$ is the orientational order parameter. $\mathrm{T}_{\mathrm{NI}}$ is typically $>60^{\circ} \mathrm{C}$ [24] while the index typically varies in the range $0.1<\beta<0.3$. The birefringence thus decreases with temperature and hence phase change will drop, however, this is a slowly changing function, except near $\mathrm{T}_{\mathrm{NI}}$ where $\Delta \mathrm{n}$ goes rapidly to zero. Using this form for $\Delta \mathrm{n}$ in equation (2) and differentiating, the phase change with temperature is,

$$
\frac{\partial \phi}{\partial \mathrm{T}}=-(4 \pi \mathrm{L} / \lambda) \Delta \mathrm{n}_{0} \beta\left(1-\mathrm{T} / \mathrm{T}_{\mathrm{NI}}\right)^{\beta-1} / \mathrm{T}_{\mathrm{NI}}
$$

Setting $\mathrm{T}=293^{\circ} \mathrm{K},\left(20^{\circ} \mathrm{C}\right), \mathrm{T}_{\mathrm{NI}}=333^{\circ} \mathrm{K}\left(60^{\circ} \mathrm{C}\right)$ and using a typical value of $\Delta \mathrm{n}=0.30$ at room temperature with $\beta=0.19$, then $\Delta \mathrm{n}_{0}=0.45\left(0^{\circ} \mathrm{K}\right)$. Using these values, equation (3) yields $\frac{\partial \phi}{\partial \mathrm{T}}=-0.01 \pi \mathrm{rad} /{ }^{\circ} \mathrm{K}$. Hence with a temperature change $\Delta \mathrm{T}=5^{\circ} \mathrm{C}$, the phase change $\delta \phi=-0.05 \pi$ radian. At $1.064 \mathrm{~nm}$, this corresponds only to $0.025 \lambda$. This very small value infers that the source of the phase response change $\delta \phi$ is more likely due to changes in the LC layer thickness $L$. As the LC layer thickness is given by $L=\lambda / 2 \Delta \mathrm{n}$, a reduction in $\pi$ phase change corresponds to an effective change in $\delta \mathrm{L}=\lambda / 4 \Delta \mathrm{n}=0.88 \mu \mathrm{m}$. This could occur through mechanical distortion of the chip structure due to the developing temperature gradient, observed experimentally and predicted above combined with differences in thermal expansion coefficients of the various materials.

Let us assume that the temperature gradient results in a change in the thickness of the LC layer, which is maximum in the centre and reducing towards the edges. This varying thickness affects the phase response so that, for a given GL, the centre produces a different phase delay than the edges. In the experiments, this phase delay is converted into a rotation of the linear polarization (see figure 2) and thus the polarization direction will vary from the centre to the edges. The effect of the polarizer (TFP in figure 2) is to transmit only the component of the polarization that is parallel to its axis. For example, the transmitted power from a uniform linearly polarized beam with polarization angle $\theta$ is $P_{t}=P_{i} \cos ^{2} \theta$.

To estimate the propensity of the temperature gradient to induce a non-uniform polarization direction, we will assume that the beam can be modelled as the sum of three concentric areas, each having a distinct uniform polarization angle. The laser power transmitted through the TFP from the central area is $P_{t 1}=P_{i 1} \cos ^{2} \theta$ whereas the laser power transmitted from the remaining two concentric areas around the centre $\mathrm{P}_{\mathrm{t} 2}$ and $\mathrm{P}_{\mathrm{t} 3}$ will be phase 
shifted by varying degrees. The normalized transmitted power is the sum of all three components. An example solution is,

$$
P_{t} / P_{i}=\left[1.3 \cos ^{2} \theta+1.3 \cos (\theta / 2+\pi / 8)^{2}+0.4(3 \theta / 4+3 \pi / 4)^{2}\right] / 3
$$

Figure 9 shows the resulting expected phase responses at low and high average powers. In figure 9(a) at average power $\langle\mathrm{P}\rangle=26.2 \mathrm{~W}$, experiment is compared with a pure $\cos ^{2} \theta$ function showing excellent agreement with experiment over $0-2 \pi$. Figure 9 (b) shows the example phase response (above) compared with the experimental data at high average power $\langle\mathrm{P}\rangle=160 \mathrm{~W}$. The fit of theory and experiment is in reasonable agreement and supports the view that the variations in LC thickness across the chip due to the developing thermal gradient may be the source of the limited phase range at high average laser powers.
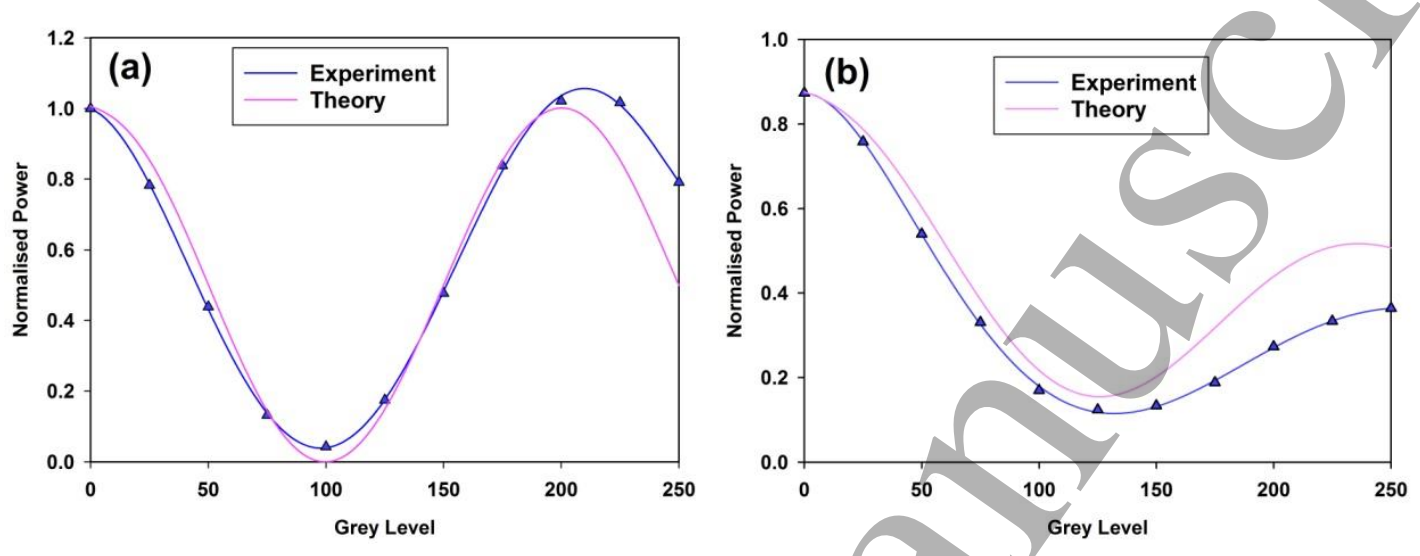

Figure 9. The calculated phase response with incident average power. (a) experimental data at low average power, $\langle\mathrm{P}\rangle=26.2 \mathrm{~W}$ compared with a pure $\cos ^{2} \theta$ function, (b) when three regions of the thermally affected region of the chip are modelled with different phase response, equation (4) and compared to experiment at $\langle\mathrm{P}\rangle=160 \mathrm{~W}$. This limited phase response will affect diffraction efficiency significantly.

\section{Laser Micro-machining results}

5.1 Parallel beam laser surface processing at $\langle\mathrm{P}\rangle \leq 25 \mathrm{~W}$

The laser mode, observed on a NIR card at low powers of a few Watts was elliptical with major axis in the horizontal. Dimensions of the raw beam were estimated to be approximately $12 \mathrm{x} 7 \mathrm{~mm}$, yielding an eccentricity $\mathrm{e} \sim 1.7$. The mode at high powers remained elliptical, confirmed by observations with a hand held NIR camera (Find-R-scope) looking at the low intensity scatter from the SLM chip and other optics. Prior to laser-micromachining with the cooled SLM, the TFP and $\lambda / 4$ plate were removed and a $4 \mathrm{f}$ optical system added with $\mathrm{f} 1=$ $300 \mathrm{~mm}$, f2 $=160 \mathrm{~mm}$ (both plano-convex, AR coated) hence reducing the beam diameter by $\mathrm{x} 0.53$. This helped to reduce the likelyhood of diffracted beams clipping the edges of any following $25 \mathrm{~mm}$ diameter optics, thus avoiding energy losses. The reflected laser output from the SLM was directed after lens L2 to the input aperture (14mm) of a digital scanning galvo (Scanlab intelliScan 14/RTC-5 card) and focussed with a $100 \mathrm{~mm}$ focal length tele-centric lens operating under Scaps GmbH scanner software. With phase CGH applied, the complex optical field at the SLM was thus re-imaged to the input aperture of the scanning galvo. A polished stainless steel plate, $60 \times 60 \times 3 \mathrm{~mm}$ was placed on an adjustable lab jack for vertical control to bring the surface to the focal plane. In addition, polished brass coated steel plates, $80 \times 80 \times 2 \mathrm{~mm}$ were exposed for highly parallel beam patterning. At a given power, the phase response was re-checked and Grey Level required for pecisely $2 \pi$ phase difference determined. This allowed one to alter the blazing function in Labview software relevant to a 
given power level. As phase CGH's can allow efficient use of the high power laser through diffractive splitting, parallel beam surface micro-processing was carried out at a range of powers.

A CGH generating three spots $( \pm 1$ and zero order) on a polished stainless steel plate were created using the non-iterative lens and gratings algorithm in Labview. Figure 10(a) shows an optical image of the three ablated parallel lines at low power with laser parameters of $10 \mathrm{kHz},\langle\mathrm{P}\rangle=2 \mathrm{~W}$ and $250 \mathrm{mms}^{-1}$ scan speed. The lines have approximately the same width $(80-100 \mu \mathrm{m})$ showing that laser power was evenly distributed between the orders. Pulse energy was therefore $\sim 70 \mu \mathrm{J}$ per pulse per beam. At the end of a line scan, the decelerating beam created an imprint of the laser intensity distribution and by fitting an ellipse to the outline in optical software, the beam spot dimensions were estimated to be $\sim 88 \pm 1 \mu \mathrm{m}$ x $31 \pm 4 \mu \mathrm{m}$ with beam ellipticity e $\sim 2.8$. However, most of the energy appeared in a central lobe, approximately $32 \pm 2 \mu \mathrm{m} \times 23 \pm 2 \mu \mathrm{m}$ giving ellipticity of the central region as e $\sim 1.4$. Averaging these yields $\mathrm{e} \sim 2.1$, a more realistic estimate and closer to the $\sim 1.7$ estimated using the NIR card. Laser beam quality factors were estimated to be $\mathbf{M}^{2}{ }_{\mathrm{X}} \sim 4.3$ and $\mathrm{M}^{2}{ }_{\mathrm{Y}} \sim 2.3$ respectively from the observed surface ablation dimensions. Single pulse fluence was therefore around $F=2.8 \mathrm{Jcm}^{-2}$ and pulse overlap $\mathrm{N} \sim 25$ per spot along the smaller axis.

Figure 10(b) shows the \pm 1 (overlapped) and zero orders machined at $1 \mathrm{~ms}^{-1}$ with $\langle\mathrm{P}\rangle=25 \mathrm{~W} / 404 \mathrm{kHz}$, hence with pulse energy $\mathrm{E} \sim 21 \mu \mathrm{J}$ and fluence $\mathrm{F} \sim 0.85 \mathrm{Jcm}^{-2}$. As offset hatch spacing was $460 \mu \mathrm{m}$, this overlapped the $\pm 1^{\mathrm{st}}$ orders, yielding an asymmetric pattern. Figure 10(c) shows an SEM image of the asymmetric pattern.
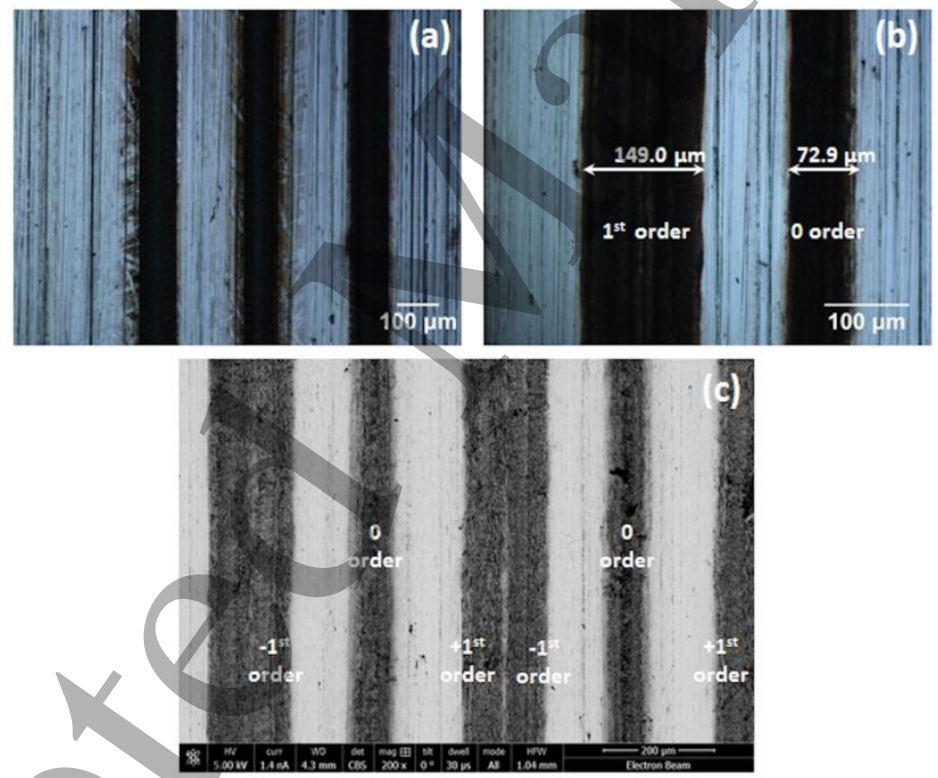

Figure 10. (a) Optical micrograph of three parallel lines ( $\pm 1^{\text {st }}$ order, $0^{\text {th }}$ order middle) machined at fluence $\mathrm{F}=2.8 \mathrm{Jcm} \mathrm{Jm}^{-2}$ on stainless steel with $\langle\mathrm{P}\rangle=2 \mathrm{~W}, 10 \mathrm{kHz}$ and scan speed $250 \mathrm{~ms}^{-1}$, separated by $250 \mu \mathrm{m}$ with line widths $\sim 90 \mu \mathrm{m}$, (b) optical image of large area 3 spot processing with $\langle\mathrm{P}\rangle=25 \mathrm{~W} / 404 \mathrm{kHz}$ exposure with $1 \mathrm{~ms}^{-1}$ scan speed and offset hatch spacing of $460 \mu \mathrm{m}$, overlapping the $\pm 1^{\text {st }}$ orders hence yielding an asymmetric pattern. Pulse energy E $\sim 21 \mu \mathrm{J}$ and fluence $\mathrm{F} \sim 0.85 \mathrm{Jcm}^{-2}$, (c) SEM image of the stainless steel surface in (b) with $\langle\mathrm{P}\rangle=25 \mathrm{~W}$ exposure clearly showing the overlapping $\pm \mathrm{1}^{\text {st }}$ orders and zero order in between .

When linearly polarised ultrashort pulses are scanned on a surface with fluence near the ablation threshold, Laser Induced Periodic Surface Structures (LIPSS) can result due to the interference of a surface scattered wave with the incoming radiation [25,26] This interference modulates the light intensity of the radiation on the surface, resulting in periodic ablation with pitch close to the wavelength of the exciting laser. Ultrashort laser pulses with $\leq 10 \mathrm{ps}$ pulselengths are ideal for encoding diffractive, periodic surface structures since heat 
diffusion and melting are minimised during ablation [27] White light is easily diffracted by these periodic surface undulations. Figure 11(a) shows the result of large area parallel beam surface ablation (3 spots) at $\langle\mathrm{P}\rangle$ $=25 \mathrm{~W}$ average power, $404 \mathrm{kHz}$ repetition rate and $3 \mathrm{~ms}^{-1}$ scan speed. The colour differences are due to the slight change in viewing angles of the camera lens. Pulse energy was hence around $21 \mu \mathrm{J}$ and average power $\langle\mathrm{P}\rangle \sim$ $8.3 \mathrm{~W}$ per beam. With focused beam area of $\sim 2.5 .10^{-5} \mathrm{~cm}^{2}$, the single pulse fluence is approximately $\mathrm{F} \sim$ $0.85 \mathrm{Jcm}^{-2}$. As the hatch spacing was $260 \mu \mathrm{m}$, the whole surface was patterned and effective patterning rate $\mathrm{R} \sim$ $8 \mathrm{~cm}^{2} \mathrm{~s}^{-1}$. Figure 11(b) clearly shows the periodic surface micro-structure (SEM) parallel to the scan direction while figure 11(c) a very high resolution SEM image of both micro and nano-structures formed within the surface relief.
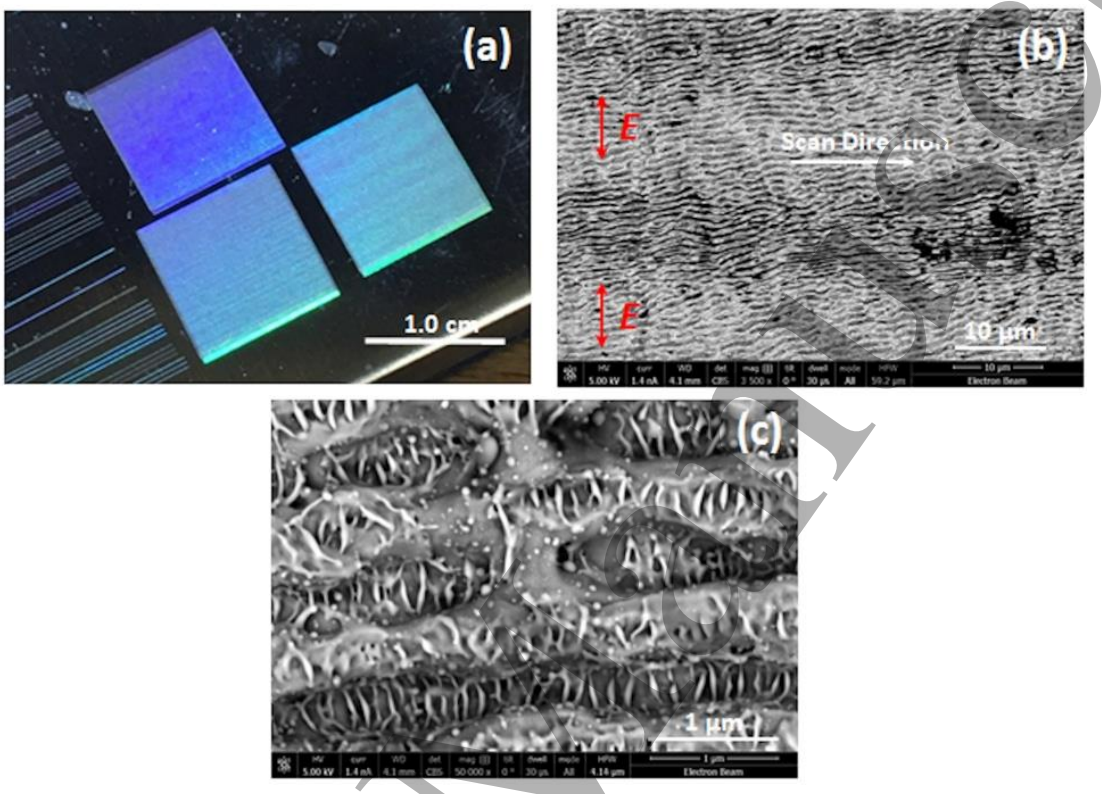

Figure 11. (a) Optical image of white light diffraction from large area $\left(1 \mathrm{~cm}^{2}\right)$ periodic structuring on stainless steel with 3 parallel spots, linearly polarized normal to scan direction. Laser exposure was $\langle\mathrm{P}\rangle=25 \mathrm{~W}, 404 \mathrm{kHz}$ with $3 \mathrm{~ms}^{-1}$ scan speed, minimising pulse overlap. (b) SEM image showing clear $1 \mu \mathrm{m}$ pitch periodic structuring at $\langle\mathrm{P}\rangle=25 \mathrm{~W}$ average power. The direction of the electric vector is shown in red. (c) Detailed high resolution SEM image showing nano-structures within the main $1 \mu \mathrm{m}$ pitch surface relief.

5.2 High Power processing at Laser powers at $\langle\mathrm{P}\rangle$ above $100 \mathrm{~W}$.

The results 3 spot $\left( \pm \mathbf{1}^{\text {st }}\right.$, zero order) multi-beam surface ablation of stainless steel and $1 \mathrm{MHz}$ repetition rate with $\langle\mathrm{P}\rangle=120 \mathrm{~W}$ and $\langle\mathrm{P}\rangle=160 \mathrm{~W}$ are shown in Figure 12 with increasing numbers of overscans (top to bottom). The asymmetric pattern, previously observed at $\langle\mathrm{P}\rangle=25 \mathrm{~W}$ is still apparent at both $\langle\mathrm{P}\rangle=$ $120 \mathrm{~W}$ and $\langle\mathrm{P}\rangle=160 \mathrm{~W}$ and structures deepen with increasing exposure, as expected. The 3 spot CGH is still effective at this power level. With 50 overscans at $\langle\mathrm{P}\rangle=160 \mathrm{~W}$, the surface has oxidised. 


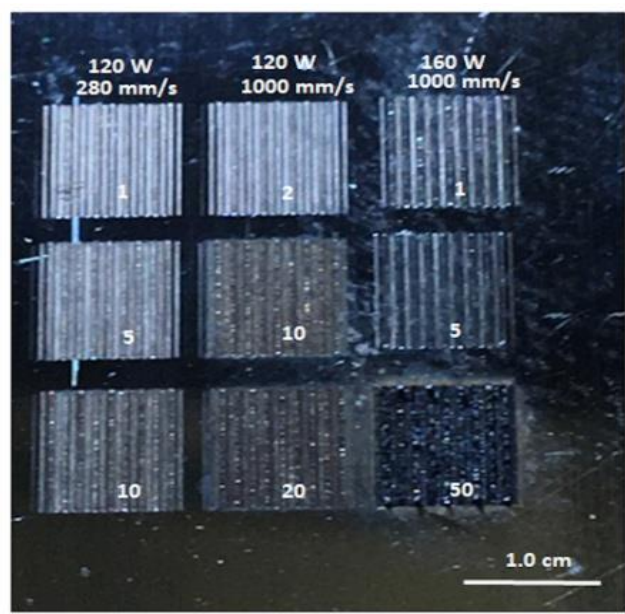

Figure 12. Optical image of three beam parallel processing with $\langle\mathrm{P}\rangle=120 \mathrm{~W} / 280 \mathrm{mms}^{-1}$ with 1,5 and 10 overscans, $\langle\mathrm{P}\rangle=$ $120 \mathrm{~W} / 1 \mathrm{~ms}^{-1}$ with 2,10 and 20 overscans, and $\langle\mathrm{P}\rangle=160 \mathrm{~W} / 1 \mathrm{~ms}^{-1}$ with 1,5 and 50 overscans. The assymetric pattern persists. With 50 overscans at $\langle\mathrm{P}\rangle=160 \mathrm{~W}$, the surface has darkened due to oxidation.

Figure 13(a) shows an SEM image of the asymmetric ablation pattern (zero order, $\pm 1^{\text {st }}$ ) machined on polished stainless steel at $\langle\mathrm{P}\rangle=160 \mathrm{~W} / 1 \mathrm{MHz} / 3 \mathrm{~ms}^{-1}$ and 1overscan confirming that the cooled SLM diffracts the radiation into the first orders, even at this extreme exposure, however with low diffraction efficiency as expected. Figure 13(b) shows an SEM image of the $\pm 1^{\text {st }}$ order ablation with $1 \mu \mathrm{m}$ pitch plasmon structuring observed at the low intensity wing.
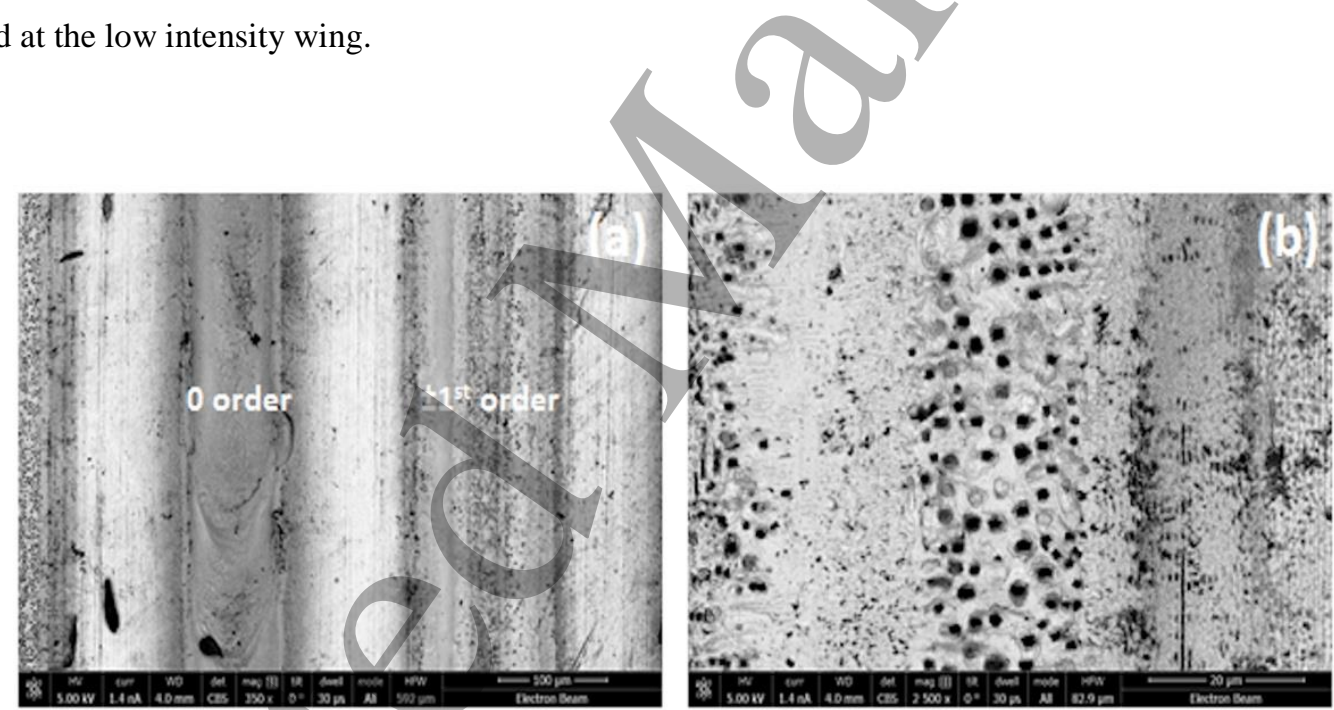

Figure 13. Parallel beam surface processing on stainless steel at $\langle\mathrm{P}\rangle=160 \mathrm{~W}$ exposure, (a) SEM image of zero and $\pm 1^{\text {st }}$ orders with scan speed $3 \mathrm{~ms}^{-1}, 1$ overscan with $\sim 0.5 \mathrm{~mm}$ offset between scans. First order diffraction efficiency is low. (b) High resolution SEM image of the overlapped $\pm 1^{\text {st }}$ orders showing surface ablation while also displaying periodic plasmon structures at the low intensity wing (RHS)

As diffraction efficiency had reduced significantly at $\langle\mathrm{P}\rangle=160 \mathrm{~W}$, large area parallel beam processing was carried out at $\langle\mathrm{P}\rangle=100 \mathrm{~W} / 404 \mathrm{kHz}$ where phase response was nearly ideal and diffraction efficiency high. A linear array of four first order rectangular intensity profiles were generated with the Hamamatsu CGH software and high speed ablation was carried out on stainless steel with a $250 \mathrm{~mm}$ F-theta lens at $20 \mathrm{~m} / \mathrm{sec}$ scan speed, figure 14. Pulse overlap was low, fluence $\mathrm{F} \sim 0.5 \mathrm{Jcm}^{-2}$ and measured ablation rate was $\mathrm{R} \sim 4 \mathrm{~mm}^{3} \mathrm{~min}^{-1}$.

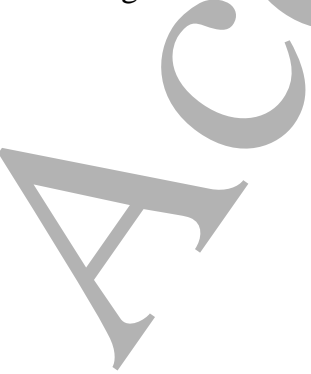




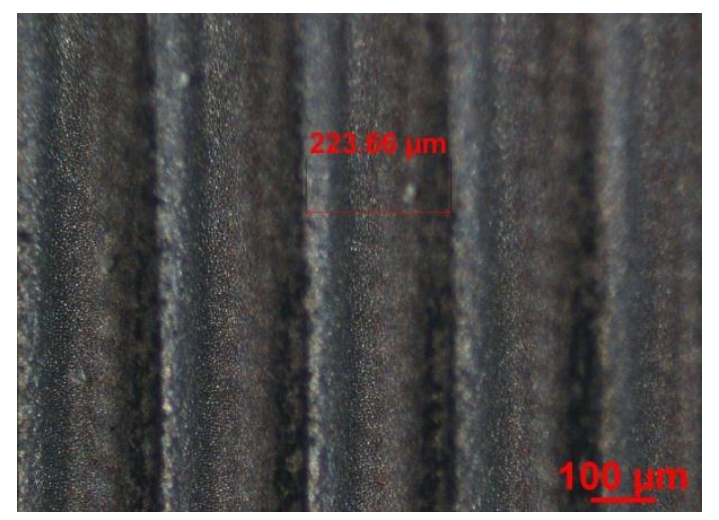

Figure 14. Microscope image of four beam (rectangular intensity) high speed large area processing of stainles steel at $\langle\mathrm{P}\rangle=$ $100 \mathrm{~W} / 404 \mathrm{kHz} / 20 \mathrm{~ms}^{-1}$ with 400 overscans at fluence $\mathrm{F} \sim 0.5 \mathrm{Jcm}^{-2}$. Ablation rate $\mathrm{R} \sim 4 \mathrm{~mm}^{3} \mathrm{~min}^{-1}$. Zero order was neglible.

5.3 More complex phase CGH's.

Patterns with 11 or more spots were next considered. Figure 15(a) shows multi spots in the form of a letter $\mathrm{M}$ pattern created in the Labview interface. The resulting complex phase $\mathrm{CGH}$, calculated by the Inverse Fourier Transform (lens and gratings algorithm) is shown in Figure 15(b). The diffracted intensity distribution with low laser power $(\langle\mathrm{P}\rangle \sim 2 \mathrm{~W})$ observed on a screen near the Fourier plane of the first lens $\mathrm{L} 1$ of the $4 \mathrm{f}$ system is shown in Figure 15(c). There is reasonable uniformity between the spot intensities observed here combined with high diffraction efficiency $\eta>90 \%$.

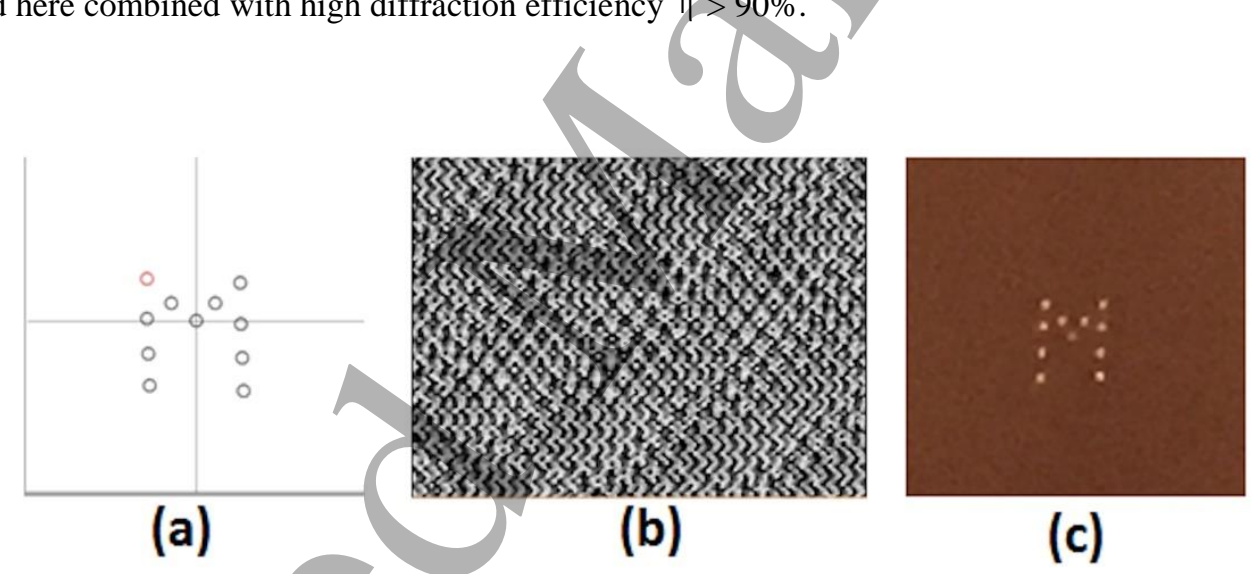

Figure 15. (a) Spot pattern set in Labvew interface representing the letter M, (b) resulting complex phase CGH created by the lens and gratings algorithm, (c) observed intensity pattern at the Fourier plane of lens L1 of the $4 \mathrm{f}$ system showing reasonable spot uniformity. Zero order spot is in the centre.

Figure 16(a-d) shows a series of letter M patterns drilled on a polished brass coated substrate with the complex phase CGH of figure 16(b) while increasing the average laser power from $\langle\mathrm{P}\rangle=90-250 \mathrm{~W}$ with temporal exposures from $10-30 \mathrm{~ms}$ at $2 \mathrm{MHz}$ repetition rate. This corresponds to 20,000 and 60,000 pulse exposure respectively. While the $\mathrm{M}$ pattern is clear at $\langle\mathrm{P}\rangle=90 \mathrm{~W}$ and $120 \mathrm{~W}$, diffraction efficiency drops significantly above these powers in accord with reduced phase response. The tails in the spots arise from the setting of the "laser delay on" in the scanner software which was non optimum, firing the laser a little too early. As the home position of the scanner was set to $(0,0)$, the tails rotate due to their positions, microstructured around $(0,0)$. 

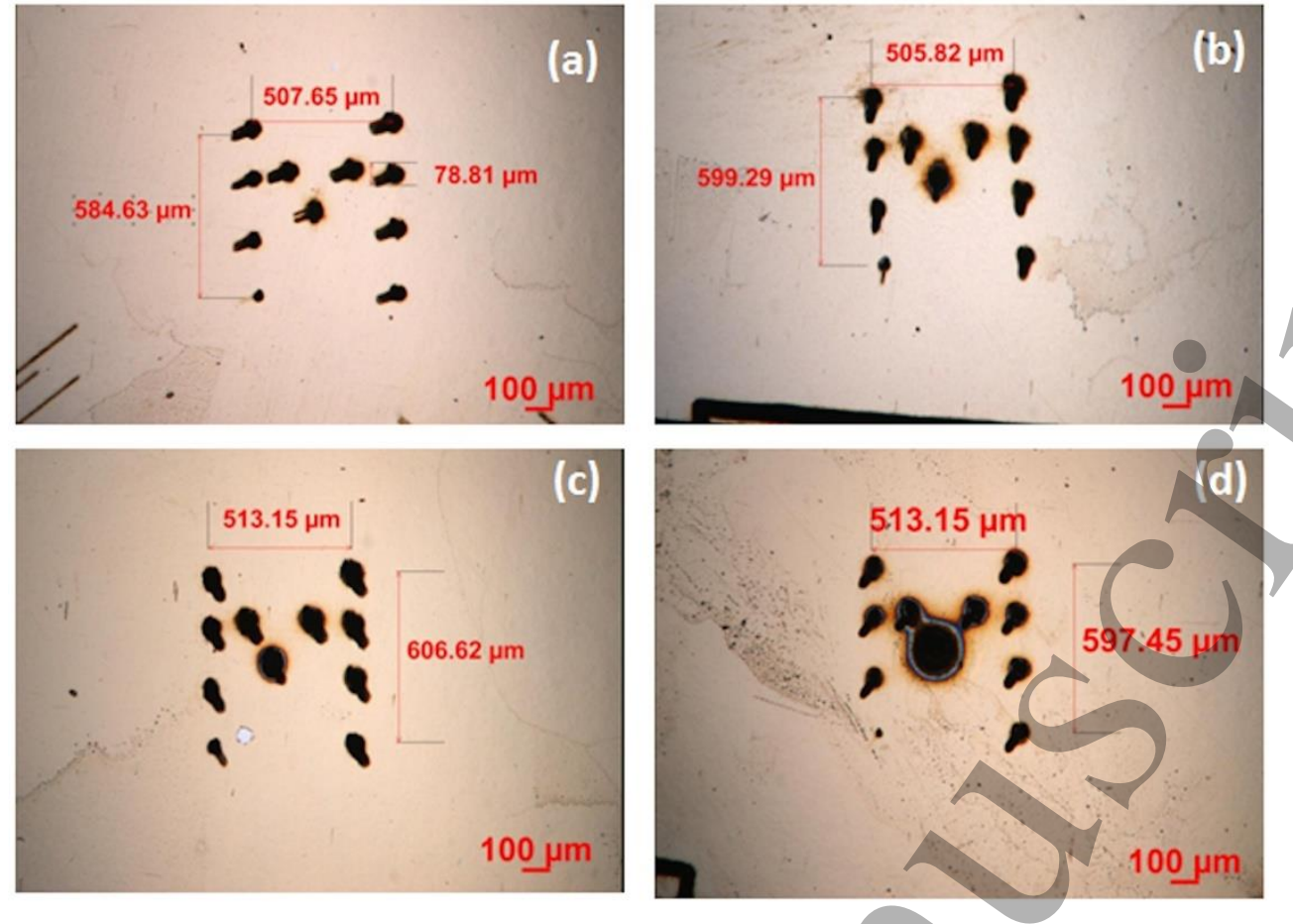

Figure 16. Optical images of surface patterning (letter "M") on polished brass plate with increasing laser powers, (a) $\langle\mathrm{P}\rangle=90 \mathrm{~W}$, $30 \mathrm{~ms},(\mathrm{~b})\langle\mathrm{P}\rangle=120 \mathrm{~W}, 30 \mathrm{~ms},(\mathrm{c})\langle\mathrm{P}\rangle=160 \mathrm{~W}, 10 \mathrm{~ms},(\mathrm{~d})\langle\mathrm{P}\rangle=250 \mathrm{~W}, 10 \mathrm{~ms}$. Diffraction efficiency drops above $\langle\mathrm{P}\rangle=120 \mathrm{~W}$ as expected.

\subsection{Long term exposure tests}

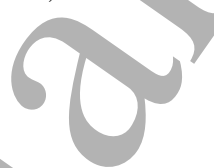

Both SLM devices have been tested for extended periods at high powers with no detrimental effects detected. In particular, continuous exposure on device- 02 for a period of 45 minutes at laser power $\langle\mathrm{P}\rangle=160 \mathrm{~W}$ and for several 20 minute exposures at $\langle\mathrm{P}\rangle=220 \mathrm{~W}$ did not result in any detectable drop in reflected power with time. No permanent change in SLM phase response characteristics have been detected during this research work.

\section{Discussion and Conclusions}

Cooled SLMs used as programmable, diffractive optics have tremendous potential for scientific and industrial applications when handling coherent radiation with high peak and average powers. The research presented in this paper, we believe, adds significantly to the contributions of Beck [14], Kaakkunen [15,16] and more recently, Klerks and Eifel [17]. Firstly, the temperature response of two efficiently cooled SLM devices (Hamamatsu X13139-03) was measured when exposed to continuous, high repetition rate, high average power $1064 \mathrm{~nm}$ picosecond laser exposure up to $\langle\mathrm{P}\rangle=220 \mathrm{~W}$. These thermal responses (measured using a thermal camera calibrated for the emissivity of Silicon) were highly linear with gradients of $0.041{ }^{\circ} \mathrm{C} / \mathrm{W}(-01)$ and 0.026 ${ }^{\circ} \mathrm{C} / \mathrm{W}(-02)$ respectively and are the first, detailed temperature data reported at multi-hundred Watt laser exposures, higher by a factor of 3.7. (at $\langle\mathrm{P}\rangle=220 \mathrm{~W}$ ) than that of Klerks and Eifal [17]. The difference in the thermal characteristics of the two test devices is likely due to a difference in thermal handling design, proprietary to Hamamatsu. A clear temperature gradient appears across the chip at laser powers above $\langle\mathrm{P}\rangle=$ $120 \mathrm{~W}$ exposure, highlighting the efficient axial heat removal. The absolute temperature rise on the best device02 with $\langle\mathrm{P}\rangle=220 \mathrm{~W}$ was only $5^{\circ} \mathrm{C}$ at the centre, posing no threat to the viability of the liquid crystal layer or silicon substrate. Thermal modelling of the SLM structure (with and without water cooling) was carried out using COMSOL multi-physics and the 3D heat diffusion equation. This model predicted that during laser exposure, the silicon chip comes to thermal equilibrium only when the SLM was water cooled, reaching $2^{\circ} \mathrm{C}$ above ambient compared to the observed $5{ }^{\circ} \mathrm{C}$ rise with $\langle\mathrm{P}\rangle=220 \mathrm{~W}$ exposure $(\langle\mathrm{P}\rangle=7 \mathrm{~W}$ absorbed). The expected chip temperature rises linearly with exposure in accord with observations and in particular, a 
temperature gradient develops at higher average powers $\langle\mathrm{P}\rangle$ above 120W. Allowing for photo-absorption in the organic LC layer, an estimated $0.4^{\circ} \mathrm{C}$ additional temperature rise could be expected. Combining these two estimates, a $2.5{ }^{\circ} \mathrm{C}$ rise is within a factor of two of the observed temperature rise.

The thermal response time of our cooled SLMs was approximately 1-2 seconds, observed with the thermal camera when cutting off the laser power at $\langle\mathrm{P}\rangle=220 \mathrm{~W}$, supporting the very efficient heat removal from the Silicon chip and consistent with the predicted response time from thermal modelling. As device reflectivity is $97 \%$, then the absorbed power $\left\langle\mathrm{P}_{\mathrm{abs}}\right\rangle \sim 7 \mathrm{~W}$ thermal load was removed efficiently at $\langle\mathrm{P}\rangle=220 \mathrm{~W}$ incident laser power.

The critical phase response with increasing exposure on the two cooled devices was measured using polarisation modulation by the SLM followed by polarisation analysis with quarter waveplate and thin film polariser (TFP). This unique approach directly generated the phase response curves (for device-01 and -02) up to $\langle\mathrm{P}\rangle=140 \mathrm{~W}$ and $\langle\mathrm{P}\rangle=200 \mathrm{~W}$ respectively. Device -02 , with the best thermal response, also showed a superior phase response, achieving full $2 \pi$ phase change at $\langle\mathrm{P}\rangle=109 \mathrm{~W}$ exposure, almost $3 \pi / 2$ radians at $\langle\mathrm{P}\rangle=160 \mathrm{~W}$, while at $\langle\mathrm{P}\rangle=200 \mathrm{~W}$, this reduced to just over a $\pi$ phase change. Hence, the operational device limit is currently at an exposure level of $\langle\mathrm{P}\rangle=130 \mathrm{~W}$. The source of the shifting phase response is likely due to liquid crystal thickness variations caused by the developing temperature gradient across the chip with increasing exposure, confirmed by the thermal camera images

The phase response was modelled theoretically, considering both the possible temperature dependence of the LC birefringence $\Delta \mathrm{n}$ and thickness of the LC layer L. As the effect of temperature on $\Delta \mathrm{n}$ appeared neglible, the phase changes thus appear to be due to variations in the LC layer thickness $\delta \mathrm{L}$ with temperature and the developing temperature gradient across the Silicon chip. This varying thickness affects the phase response so that, for a given GL, the centre produces a different phase delay (polarisation) than the edges. The beam was modelled as the sum of three concentric areas, each having a distinct uniform polarization angle and a trial solution involving the sum of these varying polarisations was compared with the observed response at $\langle\mathrm{P}\rangle=$ $160 \mathrm{~W}$ with reasonable agreement. Theory and experiment, as expected, were excellent agreement at lower average power $\langle\mathrm{P}\rangle=26 \mathrm{~W}$.

At $\langle\mathrm{P}\rangle=109 \mathrm{~W}$ exposure, the degree of polarisation remains high with $P=\left(I_{\max }-I_{\min }\right) /\left(I_{\max }+I_{\min }\right)=0.90$ while at $\langle\mathrm{P}\rangle=160 \mathrm{~W}$ and $\langle\mathrm{P}\rangle=196 \mathrm{~W}, P=0.75$ and 0.64 respectively. A spatial variation of the polarisation state across the reflected beam will occur due to the temperature gradient, hence the reduced value of $P$. While Kaakkunen [15] demonstrated cooled SLM exposure with $\langle\mathrm{P}\rangle=190 \mathrm{~W}$ continuous wave, no phase or temperature data were measured during their experiments on laser cutting which was only marginally improved over the zero order Gaussian beam. Their low diffraction efficiency can be understood from the results achieved here.

We also presented experimental results of parallel processing on polished metals with continuous powers from $25 \mathrm{~W}-160 \mathrm{~W}$ in this paper with interesting results. High speed periodic, $1 \mu \mathrm{m}$ pitch surface micro-structuring with $25 \mathrm{~W}$ average power created a continuous, diffractive surface on stainless steel, patterned at a rate of $\sim$ $8 \mathrm{~cm}^{2} \mathrm{~s}^{-1}$. Efficient, multi- beam ablation on stainless steel with up to $\langle\mathrm{P}\rangle=120 \mathrm{~W}$ was demonstrated while short (10-30ms) exposures on brass coated stainless steel substrates (11 spot $\mathrm{M}$ pattern) showed reduced diffraction efficiencies above $\langle\mathrm{P}\rangle=120 \mathrm{~W}$.

High power exposure tests were completed with continuous operation at $\langle\mathrm{P}\rangle=160 \mathrm{~W}$ for 45 minutes and at $\langle\mathrm{P}\rangle$ $=220 \mathrm{~W}$ for several 15 minute periods with no degradation observed on device-02. Total exposure times during these detailed experiments amount to many hours on both devices compared with a 2 hour test at $\langle\mathrm{P}\rangle=60 \mathrm{~W}$ by Klerks and Eifel [17]. If chip damage was going to happen, this would probably happen in seconds, not minutes, due to thermal runaway at these extreme powers. Any absorbing defect would rapidly heat and boil the liquid crystal, however, no permanent changes in liquid crystal performance have been detected in this research work. However, long term changes in diffraction efficiency with high laser exposures could yet be an issue but would require detailed measurements over much longer periods. 
One may ask what the damage threshold for irreversible changes in SLM performance will likely be. High energy pulses (fs-ns) with fluence $\mathrm{F}>0.5 \mathrm{Jcm}^{-2}$ might well result in irreversible damage to the structure as dielectric coating damage thresholds are typically at this level. Damage can also result from very high peak intensities induced by non-linear (NL) multi-photon absorption, creating absorbing defects in the liquid crystal. However, as this LCOS technology can handle I $>20 \mathrm{GWcm}^{-2}$, the effect is irrelevant here at peak powers $\mathrm{P}_{\text {peak }}$ $\sim 200 \mathrm{MWcm}^{-2}$. High average powers result in a liquid crystal chip temperature rise through absorption both in the Silicon, ceramic and copper substrates. As the absorption coefficient of pure silicon at $1064 \mathrm{~nm}$ is only $10 \mathrm{~cm}^{-}$ ${ }^{1}$ then approximately $63 \%$ of the incident radiation would be absorbed in a $1 \mathrm{~mm}$ thick-Si wafer while the transmitted radiation will be absorbed in the cooling structure below the Si chip.

In conclusion, we have demonstrated for the first time, temperature and critical phase response of /cooled SLMs exposed to average laser powers exceeding $\langle\mathrm{P}\rangle=200 \mathrm{~W}$ at $1064 \mathrm{~nm}$, a factor of 3.7 (at $\langle\mathrm{P}\rangle=220 \mathrm{~W}$ ) higher than that achieved recently by Klerks and Eifel [17], combined with $P_{\text {peak }}=200 \mathrm{MW}$ peak powers with no sign of irreversible optical damage. Modelling of the thermal and phase response has also been presented and compared with experiment. Thermal effects are due mainly to photo-absorption in the Silicon chip and the phase response limited by LC thickness variations due to the developing temperature gradient across the chip. Parallel beam micro-structuring up to $\langle\mathrm{P}\rangle=120 \mathrm{~W}$ was demonstrated on stainless steel with high diffraction efficiency, however, higher average powers result in low diffraction efficiency due to the loss of phase range in accord with expectations. The thermal and optical phase responses are highly correlated, allowing one to understand device limitations at high exposures. If the temperature gradient could be removed by further improved thermal cooling, then it may be possible to improve performance above the current operational limit of $\langle\mathrm{P}\rangle=130 \mathrm{~W}$.

Ultimately, testing these devices with both ultrahigh peak intensities exceeding $\mathrm{P}_{\text {peak }}=50 \mathrm{GWcm}^{-2}$ combined with multi hundred Watt average powers would be highly desirable. Scientific applications such as NL filamentation in air for control of lightning discharges may be possible [29] where dynamic control of phase, polarisation and orbital angular momentum of ultrahigh peak power pulses at high repetition rate would be beneficial. Future expansion of industrial applications in ultra high throughput laser marking, patterning and machining are likely when high power laser sources are combined with polygon scanners [30] able to achieve scan speeds on a substrate $\mathrm{s}>200 \mathrm{~ms}^{-1}$. When combined with a high energy, high average power laser system and optimised CGHs creating uniform or variable intensity spots [31] massively parallel-beam laser microstructuring for industrial applications will be possible.

\section{Acknowledgements}

We would like to thank Hamamatsu (Japan) for providing two X13139-03 cooled SLM devices for this collaborative research work and Hamamatsu (Germany) for loan of a 1280 x 1024 SLM controller. We are also grateful to Dr. Phil Harrison for supplying the polarisation optics and to Professor Miles Padgett of the University of Glasgow, Scotland for allowing use of the Labview software.

\section{References}

1. J. D. McKnight, D. G. Vass and R.M. Sillitto, "Development of a spatial light modulator: a randomly addressed liquid-crystalover-nMOS array," Applied Optics, Vol. 28, Issue 22, pp. 4757-4762 (1989)

2 D. J. McKnight, K. M. Johnson and R. A. Serati, "256 × 256 liquid-crystal-on-silicon spatial light modulator", Applied Optics, Vol. 33, Issue 14, pp. 2775-2784 (1994),

3 M.E. Motamedi, editor, MOEMS (Micro-optical electro-mechanical systems) SPIE Press, Bellingham, Washington (2005)

J. Courtial, K. Dholakia, L. Allen, and M. J. Padgett, "Gaussian beams with very high orbital angular momentum," Opt. Commun. 144(4-6), 210-213 (1997).

5 N. B. Simpson, K. Dholakia, L. Allen, and M. J. Padgett, "Mechanical equivalence of spin and orbital angular momentum of light: an optical spanner", Opt.Lett Vol. 22, No. 1 (1997)

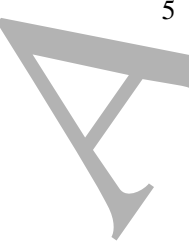


6 Y. Hayasaki, T. Sugimoto, A. Takita, N. Nishida, "Variable holographic femtosecond laser processing by use of a spatial light modulator" Applied Physics Letters, 87 p. 031101, (2005)

7 Y. Jin, O. J. Allegre, W. Perrie, K. Abrams, J. Ouyang, E. Fearon, S. P. Edwardson, and G. Dearden, "Dynamic modulation of spatially structured polarization fields for real-time control of ultrafast laser-material interactions", Optics Express vol.21, no.21 25333-25343 (2013)

8 Z. Kuang, W. Perrie, D. Liu, S.P. Edwardson and G. Dearden "Ultrafast laser parallel microdrilling using multiple annular beams generated by a spatial light modulator" J.Phys. D: Applied Physics 47, p.115501 (2014)

9 J. Ouyang, W. Perrie, O,J. Allegre, T. Heil, Y.Jin, E. Fearon, D. Eckford, S.P. Edwardson and G. Dearden "Tailored optical vector fields for ultrashort-pulse laser induced complex surface plasmon structuring" Optics Express vol. 23, no.10 12562$12572(2015)$

10 Jahja I. Trisnadi, Clinton B. Carlisle and Robert Monteverde, "Overview and applications of Grating-Light-Valve-based optical write engines for high-speed digital imaging", Proc. SPIE 5348, MOEMS Display and Imaging Systems II, 52, (January 24, 2004)

11 K. J. Mitchell, S. Turtaev, M. J.Padgett, T. Cizmar and D. J. Phillips, "High speed spatial control of the intensity, phase and polarisation of vector beams using digital micro-mirror device" Optics Express, 24(25), 29269-29282 (2016)

12 P. Schaaf, editor, "Laser Processing of Materials, Fundamentals, Applications and Developments", Springer series in Material science 139 Springer Verlag Heidelberg (2010)

13 J. Leach, G. Sinclair, P. Jordan, J. Courtial, M. J. Padgett, J.Cooper and Z. Laczik, "3D manipulation of particles into crystal structures using holographic optical tweezers" Optics Express vol.12, 220 (2004).

14 R. J. Beck, J. P. Parry, W. N. MacPherson, A. Waddie, N. J. Weston, J.D. Shephard, and D. P Hand, "Application of cooled spatial light modulator for high power nanosecond laser micromachining" Optics Express, Vol. 18, Issue 16, pp. 17059-17065 (2010)

15 J. J. J. Kaakkunen, I. Vanttaja and P. Laakso, "Fast Micromachining Using Spatial Light Modulator and Galvanometer Scanner with Infrared Pulsed Nanosecond Fiber Laser" JLMN-Journal of Laser Micro/Nanoengineering Vol. 9, No. 1, p37 (2014)

16 J. J. J. Kaakkunen, P. Laakso, and V. Kujanpää, "Adaptive multibeam laser cutting of thin steel sheets with fiber laser using spatial light modulator, Journal of Laser Applications 26,032008 (2014)

17 T. Klerks and S. Eifel, "Flexible beam shaping system for next generation of process developments in laser micro-machining", $9^{\text {th }}$ International conference on Photonic technologies, LANE 2016 (Industrial paper)

18 http://www.altechna.com

19 G.R Fowles, "Introduction to Modern Optics" (2 ${ }^{\text {nd }}$ edition), Dover Publications, New York 1975

20 N. M. Ravindra, B. Sopori, O. H. Gokce, S. X. Cheng, A. Shenoy, L. Jin, S. Aberdrabbo and Y. Chang, "Emissivity measurements and modelling of Silicon-Related Materials:An Overview" International Journal of Thermophysics Vol. 22, No.5, (Sep.2001)

21 www.crystran.co.uk

22 H.Cabrera, A.Marcano and Y.Castellanos "Absorption coefficients of nearly transparent liquids measured using thermal lens spectrometry", Condensed Matter Physics Vol. 9, No 2(46), pp. 385-389 (2006)

23 M. Marinelli, H. Zammit, F. Scudieri, S, Martellucci, F. Bloisi and L. Vicari, "Simultaneous Heat Capacity and ThermalDiffusivity Photoacoastic Measurement at Liquid-Crystal Phase Transitions" Il Nuovo Cimeto, vol.9D, no.7 (1987)

24 F. Peng, H. Cheng, S. Trepathi, R. J. Twieg and S. T. Wu, "Fast-response infrared phase modulator based on polymer network liquid crystal" Opt. Mat. Express vol.5, no.2 p265-273 (2015)

25 B Tan and K Venkatakrishnan, “ A femtosecond laser-induced periodical surface structure on crystalline silicon" J. Micromech. Microeng. (2006) 161080

26 F. Garrelie, J. P. Colombier, F. Pigeon, S. Tonchev, N. Faure, M. Bounhalli, S. Reynaud, and O. Parriaux, "Evidence of surface plasmon resonance in ultrafast laser-induced ripples" Optics Express, Vol. 19, Issue 10, pp. 9035-9043, (2011)

B. N. Chichkov, C. Momma, S. Nolte, F. von Alvensleben, A. Tünnermann "Femtosecond, picosecond and nanosecond laser ablation of Solids" Appl. Phys. A (1996) 63: 109.

28 H. Wang, X. Liu and Z. M. Zhang, 225 “Absorption Coefficients of Crystalline Silicon at Wavelengths from $500 \mathrm{~nm}$ to 1000 nm" Int. J. Thermophys (2013) 34:213-225 
29 Jerome Kasparian and Jean-Pierre Wolf "On lightning control using lasers" in Progress in Ultrafast Intense Laser science V, Springer series in Chemical Physics, chapter 6, p109, editors K. Yamanouchi, A. Guilini and K. Ledingham (Heidelberg 2009)

30 B. Jaeggia, B. Neuenschwandera , M. Zimmermann, R. De Loor and L. Penning, “ High throughput ps-laser micro machining with a synchronized polygon line scanner", 8th International Conference on Photonic Technologies LANE 2014 Industrial Paper.

31 M. Silvennoinen, J. Kaakkunen, K. Paivasaari and P. Vahimaa "Parallel femtosecond laser ablation with individually controlled intensity" Optics Express Vol. 22, No. 3, 2603 (2014) 